\title{
Communication in Repeated Network Games with Imperfect Monitoring*
}

\author{
M. Laclau ${ }^{\dagger}$
}

November 26, 2012

Job Market Paper

\begin{abstract}
I consider repeated games with private monitoring played on a network. Each player has a set of neighbors with whom he interacts: a player's payoff depends on his own and his neighbors' actions only. Monitoring is private and imperfect: each player observes his stage payoff but not the actions of his neighbors. Players can communicate costlessly at each stage: communication can be public, private or a mixture of both. Payoffs are assumed to be sensitive to unilateral deviations. The main result is that a folk theorem holds if and only if no two players have the same set of neighbors.
\end{abstract}

Keywords: communication, folk theorem, imperfect private monitoring, networks, repeated games.

JEL codes: C72, C73

*This paper is based on Chapter 3 of my Ph.D dissertation. I deeply thank my advisor Tristan Tomala for his invaluable guidance and constant encouragement. I also thank Olivier Gossner, Johannes Hörner, Frédéric Koessler, Tomasz Michalski, Nicolas Vieille, Guillaume Vigeral, Yannick Viossat and seminar audiences at the Cowles Foundation at Yale University, Ecole Polytechnique, Paris Game Theory Seminar in Institut Poincaré, Paris School of Economics and the 22nd Stony Brook Game Theory Festival. The support of the HEC foundation and of the Agence Nationale de la Recherche, under grant ANR JEUDY, ANR-10-BLAN 0112 are gratefully acknowledged.

†Yale University, 30 Hillhouse Ave., New Haven, CT 06520, USA, marie.laclau@yale.edu. 


\section{Introduction}

Consider a repeated game played on a network, where nodes represent players, and edges link neighbors. Interaction is local: a player's payoff depends only on his own and his neighbors' actions. Players observe their stage payoff only, hence monitoring is private, local and imperfect. Hence, both interaction and monitoring structures are given by the network. In addition, players can send costless messages at each stage. Communication can be private or public, that is: players can send different messages to distinct players (e.g. private emails), or they can communicate publicly with a subset of players (e.g. Carbon Copy). In the latter case, the Carbon Copy list of players is certifiable. (Players could also use Blind Carbon Copy, that is a mixture of private and public communication.) This paper circumscribes the networks for which a full folk theorem holds in this setup, i.e. under which conditions all feasible, strictly individually rational payoffs are equilibrium payoffs in the repeated game when players are sufficiently patient. The main result is that a folk theorem holds if and only if no two players have the same set of neighbors (Condition DN). For a wide class of payoff functions, I construct a perfect Bayesian equilibrium (henceforth, PBE) for the family of networks that satisfy Condition DN. Condition DN is also necessary: if it is is not satisfied, then the folk theorem does not hold.

The key to the characterization lies in understanding when communication makes it possible (i) to transmit precise information about players' deviations (detection and identification) and (ii) to coordinate players' behavior. Throughout the paper, I assume that the payoff functions are such that any unilateral deviation affects each neighbor's payoff (Assumption P). Hence, neighbors' deviations are detectable, although deviators may not be identifiable. The condition on the networks' topology for a folk theorem to hold for any payoff function that satisfies Assumption P, is that any two players must have different set of neighbors (Condition $\mathrm{DN})$. It has a simple interpretation. Assume that player $i$ detects a neighbor's deviation, and that, according to player $i$, the deviator could be either his neighbor $j$ or his neighbor $k$. The condition states that there exists another player $\ell$ who (without loss of generality) is a neighbor of $j$ but not of $k$. If indeed player $j$ is the deviator, then player $\ell$ can confirm this to player $i$, since he has also detected a unilateral deviation; whereas if player $k$ is the deviator, then $\ell$ can report to player $i$ that $j$ is innocent, since $\ell$ has not detected a deviation. Now, player $\ell$ could deviate by not reporting his information. This issue is handled by requiring deviating players to confess their deviation afterwards, that is: the equilibrium strategies are such that, each player confesses a deviation after a history in which he did deviate. ${ }^{1}$ Under unilateral deviations, if player $\ell$ does not report his information truthfully, then the player

\footnotetext{
${ }^{1}$ Close arguments can be found in Ashkenazi-Golan ([2]).
} 
who deviates first, either $j$ or $k$, will confess to player $i$.

The construction of the equilibrium strategy when Condition DN is satisfied is adapted from Fudenberg and Maskin ([12]). However, since histories are private here, several modifications must be made. In particular, a communication protocol is developed in order to identify the deviator when there is a deviation. Moreover, the punishment and reward phases have to be adapted. Indeed, Condition DN does not rule out networks for which a player, say $k$, has a single neighbor, say $\ell$. In that case, player $\ell$ might have an incentive not to report player $k$ 's deviations for which player $\ell$ is the unique monitor. However, with the same argument as before which relies on unilateral deviations, if player $\ell$ does not report player $k$ 's deviation, then player $k$ confesses. Nevertheless, it might be impossible for players other than $k$ and $\ell$ to distinguish between the two following histories: "player $k$ deviates in action at stage $t$, and his unique monitor $\ell$ truthfully reports the deviation" and "player $k$ does not deviate at stage $t$, whereas player $\ell$ lies when reporting player $k$ 's deviation." However, it is then possible to punish both players $k$ and $\ell$ since no player is a neighbor of both of them: I require player $k$ (respectively $\ell$ ) to minmax player $\ell$ (respectively $k$ ), and all player $\ell$ 's neighbors to minmax player $\ell .^{2}$ Finally, public communication also serves the purpose of coordinating players' actions. In particular, minmax strategies might be mixed, and pure actions are not monitored even by neighbors. This is an obstacle to detect deviations during punishment phases, and to provide incentives (rewards) for the minmaxing players to randomize according to the distribution given by their minmax strategies. To tackle this problem, players announce the pure actions they actually play during the punishment phase (these announcements are made simultaneously with the choice of action). Therefore, players can detect the deviations of their neighbors during the punishment phase, and can reward them accordingly thereafter.

To prove that Condition DN is also necessary for a folk theorem to hold, consider the case for which two players, say $j$ and $k$, have the same neighbors. I construct a particular payoff function for which there is a feasible and strictly individually rational payoff that is not an equilibrium payoff, no matter how little players discount the future. More precisely, the payoff function is such that there exists a common neighbor of both $j$ and $k$, say player $i$, who cannot differentiate between some deviations of players $j$ and $k$. In addition, player $i$ is unable to punish both: intuitively, player $i$ rewards player $k$ (respectively $j$ ) when he punishes player $j$ (respectively player $k$ ). This is a failure of joint rationality, following the terminology of Renault and Tomala ([30]).

\footnotetext{
${ }^{2}$ This joint punishment of players $k$ and $\ell$ implies that the reward phase has to be modified from Fudenberg and Maskin ([12]), in a way such that both players $k$ and $\ell$ have lower continuation payoffs after having been minmaxed than after the punishment phase of another player (see Section 5.2.4).
} 
Application. An application of interest is a partnership game (see [28]). Consider a partnership in which production is conducted by a team whose goal is to maintain a certain level of individual effort by its members. Each member's effort is not observable and there is moral hazard (effort is costly). Assume that each member's compensation depends on the output of a subset of members only, referred to as the direct colleagues, and this output depends on the level of effort of these members. For instance, the head of a subteam's compensation may depend on his own, his direct subordinates' and on his own chief's levels of effort: this defines a subteam. In addition, agents may communicate with each other via emails, either privately or publicly. If a member is reported by his direct colleagues, i.e. identified as exerting low effort, the group can punish him by reducing his share in the total profit, which raises other members' shares. This paper shows that coordination is sustainable if and only if any two members have a non-common colleague. In particular, the following network structures $\left(G_{1}\right.$ and $G_{2}$ ) illustrate when a folk theorem cannot hold.

Figure 1:
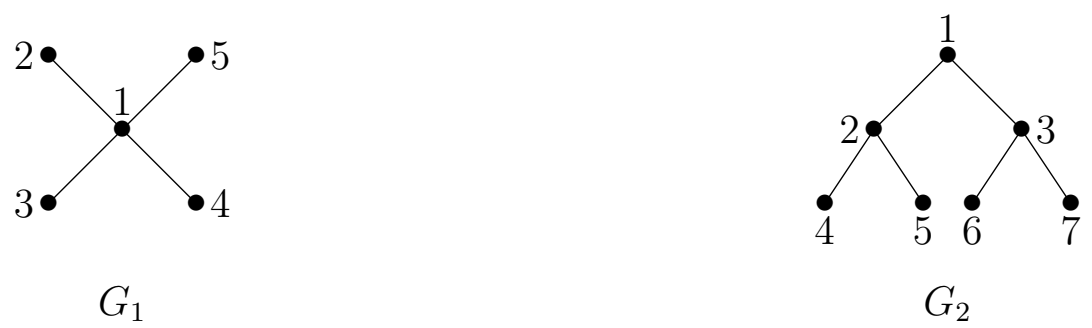

Also, no complete network satisfies Condition DN, which entails that compensation must not depend on the efforts of the whole firm's members in order to enable coordination. However, if the members form a circle (with at least five members, network $G_{3}$ in the figure below), then coordination is sustainable. Moreover, the tree structure depicted by the network $G_{4}$ also enables a folk theorem to hold.

Figure 2:

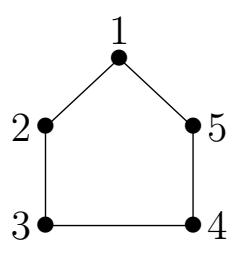

$G_{3}$

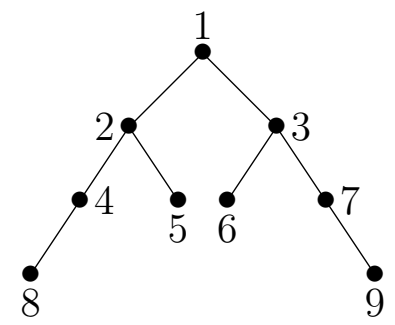

$G_{4}$ 
Related literature. This paper lies at the juncture of two independent literatures: repeated games and social networks. Regarding repeated games, the folk theorem was originally established for Nash equilibria $([4,12,31,32])$ and extended by Fudenberg and Maskin $([12,13])$ to subgame-perfect equilibria. A key assumption is perfect monitoring. A large fraction of the literature on folk theorems with imperfect monitoring has focused on imperfect public monitoring (see [11]). The model of collusion by Green and Porter ([18]) also considers imperfect public monitoring, where the market price serves as a commonly observed signal. There is a large recent literature on imperfect private monitoring (see [25] for details). Fudenberg and Levine ([10]) establish an approximate folk theorem with imperfect private monitoring without explicit communication. They consider private random signals induced by the action profile of all other players. With public communication, Compte ([8]), Kandori and Matsushima ([21]), and Obara ([27]) provide sufficient conditions for a folk theorem to hold. All three consider random signals, whereas signals are deterministic in my setup. My result departs from these folk theorems, either with private $([8,21,27])$ or public $([11])$ monitoring, in two ways. First, they consider restricted set of strategies, which enable them to characterize a set of equilibrium payoffs included (sometimes strictly) in the set of feasible and strictly individually rational payoffs. For instance, Fudenberg, Levine and Maskin ([11]) characterize the set of perfect public equilibria, which may have an empty interior. As mentioned before, my main result is on the contrary a full folk theorem. Moreover, they only provide sufficient conditions for a folk theorem to hold, whereas Condition DN is also necessary in my setup.

Closer to my setting, Ben-Porath and Kahneman ([5]) establish a necessary and sufficient condition for a folk theorem for the case in which (i) each player observes his neighbors' moves, and (ii) communication is public. Renault and Tomala ([29]) and Tomala ([34]) study undiscounted repeated games with the same signals as in [5] (i.e. each player observes his neighbors' moves) but communication is constrained by the network structure (they also establish a necessary and sufficient condition for a folk theorem to hold). All these papers $[5,29,34]$ (and also [7, 22, 26, 35]) assume that monitoring among neighbors is perfect. To the contrary, I assume here that it is imperfect: payoffs encapsulate all an agent's feedback about his neighbors' play (for instance, firms infer rivals' likely behavior from their own profits). Besides, in all these papers, interaction is global, i.e. each player interacts with all other players. Here, interactions revolve around a network. Moreover, because of Condition DN, I prove that the folk theorem even fails if the network is complete. Recently, Nava and Piccione ([26]) and Cho ([7]) study games in networks with local interaction. Yet, both assume that each player perfectly observes his neighbors' moves.

Finally, repeated games with local interaction and where players only observe their payoffs 
are studied in Laclau ([23]). This paper departs from [23] in several ways. First, in [23] communication is assumed to be private and local (i.e. players can communicate only with their neighbors). On the contrary here, communication is global (i.e. players can communicate with all opponents), and can be private or public. On the one hand, local communication seems natural in large decentralized markets, like a Hoteling competition with a large number of firms which interact and know their closest rivals only. On the other hand, global communication seems less restrictive in other applications, where players know the identity of their opponents, and are then able to communicate with all of them (via emails for instance). A good example is the above partnership game, in which it is natural to allow players to send emails to all the members of the team. Moreover, emails enable players to certify the list of receivers (Carbon Copy), hence allowing public communication among the team seems plausible.

Second, contrary to [23] where a Nash folk theorem is established, I impose sequential rationality. The main difficulty here is to provide incentives to follow the communication protocol in case of a deviation, and to follow the equilibrium strategy (in particular for the minmaxing players during the punishment phase after a deviation). The issue is that following an action deviation by some player $i$, player $j$ may deviate and send false messages. As a consequence, the content of the message " $k$ is innocent" becomes manipulable. How to update sets of innocents becomes challenging, as it is not enough to make conjectures about a single unilateral deviation, but, possibly, several consecutive deviations. The open issue is then to construct protocol that allows to identify the player who deviates in action, ${ }^{3}$ even if another player deviates during the protocol itself. As opposed to [23], the communication protocol constructed is robust to successive unilateral deviations. The main idea follows from the fact that players are required to confess their past deviations. With this strategy, a majority rule prevents a second deviator during the communication protocol to block the identification of the initial deviator. The incentives for following the equilibrium strategy are given by adapting the construction of Fudenberg and Maskin ([12]) as explained previously. This is rather surprising, since their construction crucially relies on the fact that monitoring is perfect in their setting, and is known not to be robust generically in repeated games with (private or public) imperfect monitoring. By contrast, their method can be adapted in my setup since (i) the distribution of private signals does not have full support, and (ii) public communication enables the players to learn the sequence of pure actions played by the minmaxing players during the punishment phase.

This paper is also related to the literature on social and economic networks (for an

\footnotetext{
${ }^{3}$ Recall that communication is costless, so communication deviations need not to be punished as long as they do not affect continuation play.
} 
overview of the networks literature, see Goyal, [17], and Jackson, [20]). Networks in which a player's payoff depends on his own and his neighbor's actions have been studied among others by Galeotti and al. ([15]) and Bramoullé and Kranton ([6]). However, this literature does not account for repeated games in general.

The paper is organized as follows. The model is introduced in Section 2. In Section 3, I discuss the assumption on payoff functions. The main result is stated in Section 4 . Section 5 establishes the sufficiency of Condition DN: for that purpose, I construct a communication protocol which aims at identifying the deviator when a deviation occurs; I then construct a perfect Bayesian equilibrium. Section 6 establishes that the folk theorem fails if Condition DN is not satisfied. Finally, Section 7 develops some extensions and raises open questions.

\section{The setup}

Consider a repeated game played on a fixed network where players interact only with their neighbors. This is described by the following data:

- a finite set $N=\{1, \ldots, n\}$ of players $(n \geq 3) ;^{4}$

- for each player $i \in N$, a non-empty finite set $A^{i}$ of actions (with $\sharp A^{i} \geq 2$ );

- an undirected graph $G=(N, E)$ in which the vertices are the players and $E \subseteq N \times N$ is a set of links. Let $\mathcal{N}(i)=\{j \neq i: i j \in E\}$ be the set of player $i$ 's neighbors. Since $G$ is undirected, the following holds: $i \in \mathcal{N}(j) \Leftrightarrow j \in \mathcal{N}(i)$;

- for each player $i \in N$, a payoff function of the form $g^{i}: \prod_{j \in \mathcal{N}(i) \cup\{i\}} A^{j} \rightarrow \mathbb{R}$, i.e. player $i$ 's stage payoff depends on his own and his neighbors' actions only;

- finally, a non-empty finite set $M^{i}$ of player $i$ 's messages. I assume that the cardinality of $M^{i}$ is large, the specification of the set $M^{i}$ is given in Section 5 .

I use the following notations: $A=\prod_{i \in N} A^{i}, N^{-i}=N \backslash\{i\}, A^{\mathcal{N}(i) \cup\{i\}}=\prod_{j \in \mathcal{N}(i) \cup\{i\}} A^{j}$, $a^{\mathcal{N}(i)}=\left(a^{j}\right)_{j \in \mathcal{N}(i)}$ and $g=\left(g^{1}, \ldots, g^{n}\right)$ denote the payoff vector.

Throughout the paper, the graph $G$ is assumed to be connected. Indeed, since interaction is local, players in different connected components do not interact with each other. Therefore, I model different connected components as different games.

In addition, I introduce costless communication. Players are able to communicate both privately and publicly. First, each player can send different messages to distinct players. Second, players can make public announcements to all players or to a subset of players only.

\footnotetext{
${ }^{4}$ The 2-player case reduces to perfect monitoring, see Footnote 6 in Section 3.
} 
For instance, if a player $i$ makes a public announcement to a subset $S$ of players, then the list $S$ is certifiable, that is: each player $s$ in $S$ knows that all members in $S$ received the same message (this is common knowledge among the players in $S$ ), although he does not know the messages received by players who are not in $S$. Let $M^{i}$ be a non-empty finite set of player $i$ 's messages. Let $m_{t}^{i}(j)$ represent the private message sent by player $i$ to player $j \in N$ at stage $t$, and $m_{t}^{i}(S)$ the public message sent by $i$ at stage $t$ to players in $S \subseteq N$ (hence, $m_{t}^{i}(N)$ is a public announcement to all players).

The repeated game unfolds as follows. At every stage $t \in \mathbb{N}^{*}$ :

(i) simultaneously, players choose actions in their action sets and send messages to all players, either publicly or privately as described above.

(ii) Let $a_{t}=\left(a_{t}^{i}\right)$ be the action profile at stage $t$. At the end of stage $t$, each player $i \in N$ observes his stage payoff $g^{i}\left(a_{t}^{i}, a_{t}^{\mathcal{N}(i)}\right)$. A player cannot observe the actions chosen by others, even by his neighbors.

Hence, both interaction and monitoring possibilities are given by the network $G$. In addition, I assume perfect recall and that the whole description of the game is common knowledge. For each stage $t$, denote by $H_{t}^{i}$ the set of player $i$ 's private histories up to stage $t$, that is $H_{t}^{i}=\left(A^{i} \times\left(M^{i}\right)^{N^{-i}} \times\left(M^{j}\right)_{j \in N^{-i}} \times\left\{g^{i}\right\}\right)^{t}$, where $\left\{g^{i}\right\}$ is the range of $g^{i}$ ( $H_{0}^{i}$ is a singleton). An element of $h_{t}^{i}$ is called an $i$-history of length $t$. An action strategy for player $i$ is denoted by $\sigma^{i}=\left(\sigma_{t}^{i}\right)_{t \geq 1}$ where for each stage $t, \sigma_{t}^{i}$ is a mapping from $H_{t-1}^{i}$ to $\Delta\left(A^{i}\right)$ (where $\Delta\left(A^{i}\right)$ denotes the set of probability distributions over $A^{i}$ ). A communication strategy for player $i$ is denoted by $\phi^{i}=\left(\phi_{t}^{i}\right)_{t \geq 1}$ where for each stage $t, \phi_{t}^{i}$ is a mapping from $H_{t-1}^{i}$ to $\Delta\left(\left(M^{i}\right)^{N^{-i}}\right)$. Note that a player may deviate from $\sigma^{i}$ or from $\phi^{i}$, thus I shall distinguish between action and communication deviations accordingly. A behavior strategy of a player $i$ is a pair $\left(\sigma^{i}, \phi^{i}\right)$. Let $\Sigma^{i}$ be the set of player $i$ 's action strategies and $\Phi^{i}$ his set of communication strategies. I denote by $\sigma=\left(\sigma^{i}\right)_{i \in N} \in \prod_{i \in N} \Sigma^{i}$ the players' joint action strategy and by $\phi=\left(\phi^{i}\right)_{i \in N} \in \prod_{i \in N} \Phi^{i}$ their joint communication strategy. Let $H_{t}$ be the set of histories of length $t$ that consists of the sequences of actions, payoffs and messages for $t$ stages. Let $H_{\infty}$ be the set of all possible infinite histories. A profile $(\sigma, \phi)$ defines a probability distribution, $\mathbb{P}_{\sigma, \phi}$, over the set of plays $H_{\infty}$, and I denote $\mathbb{E}_{\sigma, \phi}$ the corresponding expectation. I consider the discounted infinitely repeated game, in which the overall payoff function of each player $i$ in $N$ is the expected sum of discounted payoffs. That is, for each player $i$ in $N$ :

$$
\gamma_{\delta}^{i}(\sigma, \phi)=\mathbb{E}_{\sigma, \phi}\left[(1-\delta) \sum_{t=1}^{\infty} \delta^{t-1} g_{t}^{i}\left(a_{t}^{i}, a_{t}^{\mathcal{N}(i)}\right)\right]
$$

where $\delta \in[0,1)$ is a common discount factor. Let $\mathcal{G}_{\delta}(G, g)$ be the $\delta$-discounted game. 
The solution concept is perfect Bayesian equilibrium (PBE from now on). While there is no agreed upon definition of what restrictions this involves after histories off the equilibrium path, this plays no role in my construction, and any definition will do. The reader is referred to the definition given by Gibbons (Chapter 4 in [?]). ${ }^{5}$ In fact, I only specify strategies after private histories along which only unilateral deviations, if any, have taken place. In addition, the construction has the property that, after such histories, the specified play is optimal no matter what beliefs a player holds about his opponents' play, provided that the beliefs are such that: for every player $i \in N$, if player $i$ observes a private history compatible with a history along which no deviation has taken place (respectively along which only unilateral deviations have taken place), then player $i$ believes that no deviation has taken place (respectively only unilateral deviations have taken place). Plainly, this suffices to ensure optimality. Given that play after other histories (i.e., histories that involve simultaneous deviations) is irrelevant, the partial strategy and beliefs that I define can be completed in any arbitrary fashion. Details are given in Section 5.2.

Let $E_{\delta}(G, g)$ its associated set of PBE payoffs. For each $a \in A$, I denote $g(a)=$ $\left(g^{1}\left(a^{1}, a^{\mathcal{N}(1)}\right), \ldots, g^{n}\left(a^{n}, a^{\mathcal{N}(n)}\right)\right)$ and $g(A)=\{g(a): a \in A\}$. Let $\operatorname{co} g(A)$ be the convex hull of $g(A)$, which is the set of feasible payoffs. Player $i$ 's (independent) minmax level is defined by: 6

$$
\underline{v}^{i}=\min _{x^{\mathcal{N}(i)} \in \prod_{j \in \mathcal{N}(i)}} \max _{\left(A^{j}\right)} g_{x^{i} \in \Delta\left(A^{i}\right)} g^{i}\left(x^{i}, x^{\mathcal{N}(i)}\right) .
$$

I normalize the payoffs of the game such that $\left(\underline{v}^{1}, \ldots, \underline{v}^{n}\right)=(0, \ldots, 0)$. I denote by $I R^{*}(G, g)=$ $\left\{g=\left(g^{1}, \ldots, g^{n}\right) \in \mathbb{R}^{n}: \forall i \in N, g^{i}>0\right\}$ the set of strictly individually rational payoffs. Finally, let $V^{*}=\operatorname{co} g(A) \cap I R^{*}(G, g)$ be the set of feasible and strictly individually rational payoffs.

The aim of this paper is to characterize the networks $G$ for which a folk theorem holds, that is: each feasible and strictly individually rational payoff is a PBE payoff for all discount factors close enough to one. In the next section, I display and motivate the class of payoff functions I consider.

\footnotetext{
${ }^{5}$ Fudenberg and Tirole ([14]) give a definition for games with observable actions, which my game does not have. A recent definition for general games, including games with imperfect monitoring, is given by González-Díaz and Meléndez-Jiménez ([16]).

${ }^{6}$ It is sometimes possible here to drive equilibrium payoffs below this bound, see Section 7.
} 


\section{A class of payoff functions}

The following example is taken from [23]. It shows that a necessary condition for a folk theorem is that the payoff functions are sufficiently rich to enable players to detect deviations.

Example 3.1. Consider the 5-player game played on the following network:

Figure 3:

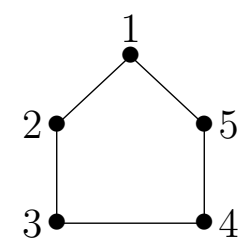

$G_{1}$

The action sets of the players are $A^{1}=A^{2}=A^{3}=\{C, D\}$ and $A^{4}=A^{5}=\{0,1\}$. The payoff functions of players 1, 2 and 3 are given by the following matrices (player 2 chooses the row, player 1 the column, and player 3 the matrix):

3 plays $C$

\begin{tabular}{|c|c|c|}
\hline $2 \backslash 1$ & $C$ & $D$ \\
\hline$C$ & $1,0,2$ & $0,0,2$ \\
\hline$D$ & $1,2,0$ & $0,6,0$ \\
\hline
\end{tabular}

3 plays $D$

\begin{tabular}{|c|c|c|}
\hline $2 \backslash 1$ & $C$ & $D$ \\
\hline$C$ & $0,0,6$ & $1,0,6$ \\
\hline$D$ & $0,2,0$ & $1,6,0$ \\
\hline
\end{tabular}

In addition, the payoff functions of players 4 and 5 are $g^{4}\left(a^{3}, a^{4}, a^{5}\right)=a^{4}+a^{5}-1$ and $g^{5}\left(a^{1}, a^{4}, a^{5}\right)=2 a^{4}+a^{5}-1$. Notice that int $V^{*} \neq \emptyset$. The minmax level of each player $i \in N$ is $\underline{v}^{i}=0$. Therefore, the payoff vector $(1,1,1,1,2)$ is feasible and strictly individually rational. Notice also that the only way the get this payoff vector is that player 2 chooses between $C$ and $D$ with probability $\frac{1}{2}-\frac{1}{2}$, that players 1 and 3 choose action $D$, and that players 4 and 5 choose action 1 . Moreover, playing 1 is a dominant strategy for both players 4 and 5 .

However, $(1,1,1,1,2)$ is not a Nash equilibrium payoff of the repeated game: for any discount factor $\delta \in[0,1)$, either player 1 or player 3 has an incentive to deviate by choosing between $C$ and $D$ with probability $\frac{1}{2}-\frac{1}{2}$. The key argument is that both deviations induce the same distribution of signals for player 2. First, player 2's payoff is 1 if players 1 and 3 take the same action, 0 otherwise. Hence, the deviations of players 1 and 3 induce the 
same distribution of payoffs for player 2, and that for every strategy (possibly mixed) of the non-deviating players. ${ }^{7}$ Hence, player 2 cannot infer the identity of the deviator from the observation of his payoffs. Second, neither player 4 nor player 5 has relevant information on the actions chosen by players 1 and 3. As a consequence, player 2 cannot identify the deviator. In addition, player 2 cannot punish both players 1 and 3: punishing player 1 requires player 2 to play $C$, in which case player 3 can get a payoff of $6 .^{8}$. On the contrary, punishing player 3 requires player 2 to play $D$, in which case player 1 can get a payoff of 6 . As a consequence, either "player 1 always plays $C$ and $D$ with probability $\frac{1}{2}-\frac{1}{2}$ and pretends that player 3 does" is a profitable deviation for player 1, or "player 3 always plays $C$ and $D$ with probability $\frac{1}{2}-\frac{1}{2}$ and pretends that player 1 does" is a profitable deviation for player $3 .^{9}$ Therefore, the outcome $(1,1,1,1,2)$ is not a Nash equilibrium payoff of the repeated game and the folk theorem fails.

On the other hand, let $g^{1}, g^{2}, g^{3}$ and $g^{4}$ remain unchanged, and assume that player 5's payoff is the following:

$$
g^{5}\left(a^{1}, a^{4}, a^{5}\right)= \begin{cases}2 a^{4}+a^{5}-1 & \text { if } a^{1}=C \\ 2 a^{4}+a^{5}-1+\epsilon & \text { if } a^{1}=D\end{cases}
$$

with $\epsilon>0$. Player 5 is now able to detect player 1's deviations. Therefore, if player 2 detects either player 1's or player 3's deviation, he can obtain from player 5 the name of the deviator (player 5 clears player 1 if he does not detect any deviation, and clears player 3 otherwise). However, player 5 could lie about the identity of the deviator. I circumvent this issue by requiring a deviating player to confess thereafter. Hence, if player 5 lies, then the initial deviator, say player 1, confesses, and player 3 claims his innocence. A majority rule then gives the name of the deviator. For a large enough discount factor, it is possible to construct a perfect Bayesian equilibrium with payoff vector $(1,1,1,1,2)$ (see Section 5 for the construction).

The previous example shows that if a deviation of a player $i$ does not alter all his neighbors' payoffs, then it may possible for some feasible and strictly individually rational payoffs not to be (Nash) equilibrium payoffs of the repeated game. ${ }^{10}$ Hence, it is not possible to get a

\footnotetext{
${ }^{7}$ Following the terminology of Aumann and Maschler (1966, re-edited in 1995, [3]), this is a jointly controlled lottery over player 2's payoffs.

${ }^{8}$ Formally, this argument does not rule out that there exists a strategy that trades off punishing the two players in a way that drives both of their payoffs below 1 ; however, it is not hard to show that this is already too much to ask; the details can be found in Section 6 .

${ }^{9}$ These arguments are formally developed in Section 6.

${ }^{10}$ For a similar phenomenon, see [24].
} 
folk theorem for all payoff functions $g$. I introduce the following assumption. ${ }^{11}$

Assumption PAYOFFS $(\mathbf{P})$. For each player $i \in N$, each neighbor $j \in \mathcal{N}(i)$, every actions $b^{j}, c^{j} \in A^{j}$ such that $b^{j} \neq c^{j}, a^{i} \in A^{i}, a^{\mathcal{N}(i) \backslash\{j\}} \in A^{\mathcal{N}(i) \backslash\{j\}}$ :

$$
g^{i}\left(a^{i}, a^{\mathcal{N}(i) \backslash\{j\}}, b^{j}\right) \neq g^{i}\left(a^{i}, a^{\mathcal{N}(i) \backslash\{j\}}, c^{j}\right) .
$$

Example 3.2. The following payoff functions satisfy Assumption P:

- for each player $i$ in $N$, let $A^{i} \subset \mathbb{N}$ and $g^{i}\left(a^{i}, a^{\mathcal{N}(i)}\right)=f\left(\sum_{j \in \mathcal{N}(i) \cup\{i\}} a^{j}\right)$ with $f$ strictly monotone;

- for each player $i$ in $N$, let $A^{i} \subset \mathbb{R}$ and $g^{i}\left(a^{i}, a^{\mathcal{N}(i)}\right)=\sum_{j \in \mathcal{N}(i)} a^{j}-a^{i}$ (this game can be seen as a generalized prisoner's dilemma for $n$ players);

- firms' profits in Cournot games;

- more generally, for each player $i$ in $N$, let $A^{i} \subset \mathbb{R}$ and $g^{i}$ strictly monotone with respect to each argument.

In the next section, I introduce a necessary and sufficient condition on the networks for a folk theorem to hold.

\section{The main result}

Theorem 4.1. The following statements are equivalent:

1. For each payoff function $g$ that satisfies Assumption $P$ and such that the interior of $V^{*}$ is nonempty, for any payoff $v$ in $V^{*}$, there exists $\bar{\delta} \in(0,1)$ such that for all $\delta \in(\bar{\delta}, 1)$, $v$ is a PBE vector payoff of the $\delta$-discounted game.

2. The graph $G$ is such that, for every pair of players $i, j \in N, i$ and $j$ have different set of neighbors (Condition DN for Different Neighbors), that is:

$$
\forall i, j \in N, \quad \mathcal{N}(i) \backslash\{j\} \neq \mathcal{N}(j) \backslash\{i\}
$$

Assume that Condition DN is satisfied, that Assumption $\mathrm{P}$ holds, and that $V^{*}$ is of full dimension. Then, for any $v \in V^{*}$, it is possible to construct a PBE with payoff $v$ for a discount factor close to one. I construct this strategy in Section 5. Intuitively, Condition DN

\footnotetext{
${ }^{11}$ This assumption implies that the two-player case reduces to perfect monitoring.
} 
makes it possible to construct a communication protocol that enables players to identify the deviator when a (unilateral) deviation occurs.

In addition, Condition DN prevents the network from having too many links, since it may lead to less information. The idea is that too many links increase the likelihood for two players to have the same neighbors, which violates Condition DN. If two players have the same neighbors, their deviations may not be distinguishable, and I exhibit particular payoff function for which the folk theorem fails (see Section 6 for the general proof). In particular, the folk theorem fails for complete networks: for some payoff functions satisfying Assumption $\mathrm{P}$, the issue is that a deviation of any player is detected when then network is complete, but every player suspects everybody, and identification of the deviator may not be possible. This is an obstacle for a folk theorem to hold, see Section 6 for details.

Now, I show some properties of the networks satisfying Condition DN. First, I display some examples of graphs that satisfy Condition DN (Figure 4), and some that do not (Figure $5)$. Notice that Condition DN is not monotonic with respect to the number of links, contrary to connectivity. For instance, both $G_{9}$ and $G_{10}$ are 2-connected, whereas only $G_{9}$ satisfies Condition DN. Also, neither $G_{3}$ nor $G_{4}$ are 2-connected, whereas only $G_{3}$ satisfies Condition DN.

Figure 4: Networks satisfying DN

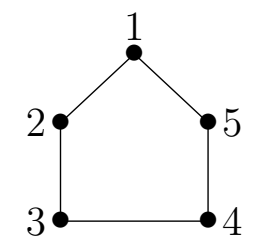

$G_{1}$

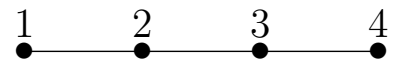

$G_{3}$

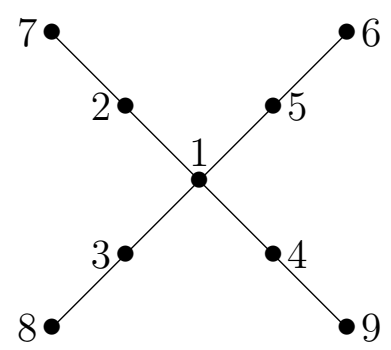

$G_{5}$
Figure 5: Networks not satisfying DN

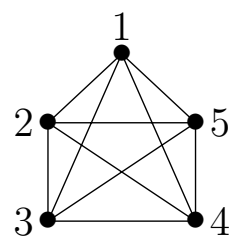

$G_{2}$

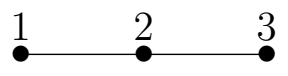

$G_{4}$

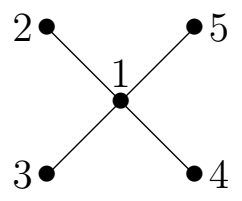

$G_{6}$ 


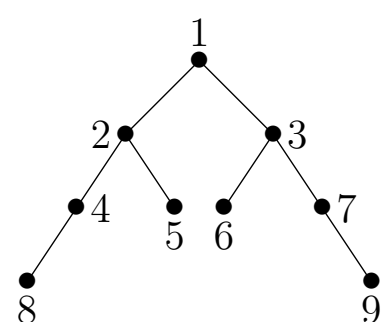

$G_{7}$

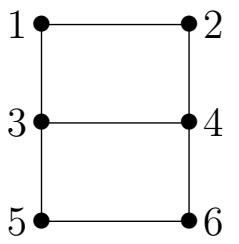

$G_{9}$

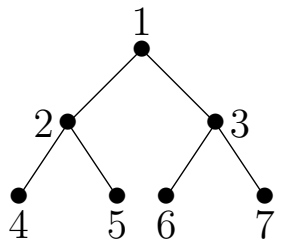

$G_{8}$

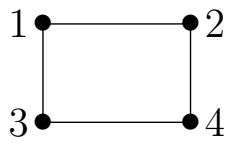

$G_{10}$

Remark 4.2. Assume that the network $G$ is connected and satisfies Condition DN. It is then easy to see that a player cannot be the unique neighbor of several players: for every players $j$ and $j^{1}$ such that $\mathcal{N}\left(j^{1}\right)=\{j\}$, and every $\ell \in \mathcal{N}(j)$ so that $\ell \neq j^{1}$, player $\ell$ has at least two neighbors. One can then easily check that a tree ${ }^{12}$ satisfies Condition DN if and only if the distance between any two terminal nodes is greater than or equal to three. Hence, no star satisfies Condition DN.

Going back to Figures 4 and 5 , the distance between two terminal nodes in $G_{7}$ is at least three, whereas the distance between nodes 4 and 5 in $G_{8}$ (or between 6 and 7) equals two.

\section{Sufficiency of Condition DN}

In this section, I assume that the network $G$ satisfies Condition DN of Theorem 4.1, namely: for any pair of players $i, j$ in $N, i$ and $j$ have at least one different neighbor. Take a payoff function that satisfies Assumption $\mathrm{P}$ and such that int $V^{*}$ is non-empty (this is assumed throughout this section). I take a point $v=\left(v^{1}, \ldots, v^{n}\right)$ in $V^{*}$ and I construct a PBE of the repeated game $\left(\sigma^{*}, \phi^{*}\right)$ with payoff $v$ for a large enough discount factor.

First of all, notice that each player $i$ detects an action deviation from a pure action profile if and only if he observes a change in his stage payoff (Assumption P). Therefore, there is an action deviation from a pure action profile of some player $k$ at stage $t$ if and only if all the

\footnotetext{
${ }^{12}$ Recall that a tree is a connected graph without cycles, and that the distance in a graph between two nodes $i$ and $j$ is the length of the shortest path from $i$ to $j$ (the reader is referred to [9] for usual definitions of graph theory).
} 
neighbors of player $k$ detect it stage $t$. Nevertheless, player $k$ 's neighbors may not be able to identify the deviator.

I first introduce a communication protocol which identifies the deviator when there is a deviation (Section 5.1). Then, I construct the equilibrium strategy (Section 5.2), and prove the equilibrium property (Section 5.3).

\subsection{Identifying a deviator}

In this section, I construct a communication protocol which identifies the deviator when there is a deviation. Then, I exhibit some properties of this protocol.

\subsubsection{Construction of the communication protocol}

Here, I assume that the players coordinate on playing a sequence on pure actions, $\bar{a}_{t}=$ $\left(\bar{a}_{t}^{i}\right)_{i \in N}$ at each stage $t>0$, and as long as no deviation is detected, on sending the message $\bar{m}_{t}(N)=\left(\bar{m}_{t}^{i}(N)\right)$, where for each player $i \in N, \bar{m}_{t}^{i}(N)=\mathcal{N}(i) \cup\{i\}$. This message means that player $i$ did not deviate, and did not detect any action deviation at stage $t-1$. Hence, according to player $i$, his neighbors and himself are innocent regarding any possible action deviation at stage $t-1$. From now on, I assume that the network $G$ is such that Condition DN holds, and that $g$ satisfies Assumption P.

We aim at identifying the deviator when a deviation occurs. Like in Example 3.1, it is not always possible to punish several players, and the identification of the deviator may therefore be needed. Nevertheless, for the family of networks that satisfy Condition DN, it is not always possible to identify the deviator. Take for instance a player $k$ who has a single neighbor $\ell$. It may be impossible for players $i \neq k, \ell$ to differentiate between the two following deviations:

- player $k$ deviates in action at stage $t$ and does not confess any deviation regarding stages $t$ and $t+1$;

- player $\ell$ deviates in communication at stage $t+1$ and claims that player $k$ deviated at stage $t$.

These deviations still have to be punished, otherwise either player $\ell$ or player $k$ might have an incentive to deviate. Yet, this is not an obstacle for a folk theorem to hold. Indeed, no player is a neighbor of both players $k$ and $\ell$, hence it is possible to minmax both players $k$ and $\ell$ : while being minmaxed, player $\ell$ is prescribed to minmax player $k$. Therefore, if one of these deviations occurs, players only have to know that the deviator is either $k$ or $\ell$. 
I then construct a communication protocol, that is: a profile of communication strategies and an output rule for each player. In my context, the communication protocol starts as soon as there is a (unilateral) deviation (either from $\bar{a}_{t}$, or from $\bar{m}_{t}(N)$ ), and does not stop before players find out the identity of the deviator (at least when it is an action deviation), or at least a subset of two players, $k$ and $\ell$, containing the deviator when the situation described above appears.

Each player $i \in N$ starts the protocol every time he detects a deviation from $(\bar{a}, \bar{m}(N))$. Players may start the communication protocol at different stages. Indeed, consider the situation where there is an action deviation of some player $j$ at stage $t$. Player $j$ 's neighbors start the protocol at the end of stage $t$, whereas other players may not start it before the end of stage $t+1$, when they receive messages from player $j$ 's neighbors. I now construct the communication protocol.

The message space. All players communicate using the same finite set of messages $\tilde{M}=$ $2^{N}$, with $N$ the set of players.

The strategy of player $i$. Player $i$ always takes the action $\bar{a}_{t}^{i}$ when he performs the protocol. ${ }^{13}$ I denote by $\tilde{\phi}^{i}$ his communication strategy during the communication protocol, which consists in announcing sets of innocents publicly to all players as follows:

- if player $i$ detects an action deviation at stage $t$, then he announces $\tilde{m}_{t+1}^{i}(N)=N \backslash \mathcal{N}(i)$ at stage $t+1$ : player $i$ claims that all his neighbors are suspects regarding a deviation at stage $t$; or, in other words, that all other players, including himself, are innocent.

- If player $i$ deviates in action at stage $t$, then he announces $\tilde{m}_{t+1}^{i}(N) \supseteq N^{-i}$ at stage $t+1$ : player $i$ confesses at stage $t+1$ that he deviated at stage $t$.

The output rule. Denote by $X_{t+1}^{i} \subset N$ player $i$ 's set of suspected players at stage $t+1$ regarding a possible deviation at stage $t$. For each player $i$ in $N$, the set $X_{t+1}^{i}$ is computed as follows.

(i) Only announcements of the form $m_{t+1}^{j}(N)=\mathcal{N}(j) \cup\{j\}, m_{t+1}^{j}(N)=N \backslash \mathcal{N}(j)$ or $m_{t+1}^{j}(N)=N^{-j}$ for each player $j \in N$ are taken into account. Other announcements are disregarded: in particular, private messages are ignored. Notice also that player $i$ takes into account his own announcement $m_{t+1}^{i}(N)$.

(ii) For every player $j$ such that $\sharp \mathcal{N}\left(j^{1}\right) \geq 2$ for each $j^{1} \in \mathcal{N}(j)$ :

\footnotetext{
${ }^{13}$ In the construction of the equilibrium strategy in the next section, player $i$ keeps playing the action corresponding to the phase of the game, instead of playing $\bar{a}_{t}^{i}$ (see Section 5.3).
} 
- if there exist at least two players $j^{1}$ and $j^{2}$ such that $j^{1} \neq j^{2}, j \in m_{t+1}^{j^{1}}(N)$ and $j \in m_{t+1}^{j^{2}}(N)$, then $j \notin X_{t+1}^{i}$ (i.e. $j$ is cleared);

- otherwise, $j \in X_{t+1}^{i}$ (i.e. $j$ is identified as suspect).

(iii) For every pair of players $k$ and $\ell$ such that $\mathcal{N}(k)=\{\ell\}$ :

- if there exist at least two players $k^{1}$ and $k^{2}$ such that $k^{1} \neq k^{2}, k \in m_{t+1}^{k^{1}}(N)$ and $k \in m_{t+1}^{k^{2}}(N)$ :

- if there exist at least two players $\ell^{1}$ and $\ell^{2}$ such that $\ell^{1} \neq \ell^{2}, \ell \in m_{t+1}^{\ell^{1}}(N)$ and $\ell \in m_{t+1}^{\ell^{2}}(N)$, then $\ell \notin X_{t+1}^{i}$ (i.e. $\ell$ is cleared);

- otherwise, $\ell \in X_{t+1}^{i}$ (i.e. $\ell$ is identified as suspect);

- otherwise, $\{k, \ell\} \subseteq X_{t+1}^{i}$ (i.e. both $k$ and $\ell$ are identified as suspects).

I now introduce an example to show how this protocol works.

Example 5.1. Consider the 4-player game played on the following network:

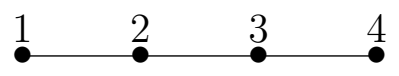

for which Condition DN is satisfied. In addition, take a payoff function $g$ for which Assumption P holds.

1. Assume first that player 2 deviates in action at some stage $t$ and, for simplicity, does not deviate in communication at stage $t$ (but possibly at stage $t+1$ ). Hence, each player $i$, except possibly 2, announces publicly $\bar{m}_{t}^{i}(N)=\mathcal{N}(i) \cup\{i\}$ at stage $t$. At stage $t+1$, players 1 and 3 should start the protocol, and stick to actions $\bar{a}_{t}^{1}$ and $\bar{a}_{t}^{3}$ respectively. In addition, strategies $\tilde{\phi}_{t+1}^{1}$ and $\tilde{\phi}_{t+1}^{3}$ prescribe to announce publicly $m_{t+1}^{1}(N)=N \backslash \mathcal{N}(1)=\{1,3,4\}$ and $m_{t+1}^{3}(N)=N \backslash \mathcal{N}(3)=\{1,3\}$ respectively. Player 4 starts the protocol at the end of stage $t+1$ only, and should announce $m_{t+1}^{4}(N)=\bar{m}_{t+1}^{4}=\{3,4\}$. Finally, player 2 should announce $\{1,3,4\}$ publicly to all players at stage $t+1$. Under unilateral deviations, players 1, 3 and 4 appear in the public announcements at stage $t+1$ of at least two different players, hence each player $i$ clears players 1, 3 and 4. Moreover, player 2 appears in at most one public announcement, therefore each player $i$ identifies player 2 as the deviator.

2. Consider now the case in which there is no action deviation at stage $t$ but a communication deviation of player 2 at stage $t+1$ who announces $m_{t+1}^{2}(N)=N \backslash \mathcal{N}(2)=\{2,4\}$ publicly to all players (recall that private messages are not taken into account in the 
strategy constructed). Under $\phi^{*}$, players 1,3 and 4 announce respectively $\{1,2\}$, $\{2,3,4\}$ and $\{3,4\}$ publicly to all players at stage $t+1$ since by assumption there is no action deviation at stage $t$. Hence, $X_{t}^{i}=\{1,2\}$ for each player $i$ in $N$. The two following cases are then possible.

- Player 2 also deviates in action at stage $t+1$. At stage $t+2$, players $1,2,3$ and 4 should announce respectively $\{1,3,4\},\{1,3,4\},\{1,3\}$ and $\{3,4\}$ publicly to all players. Even if one player deviates at stage $t+2$, each player clears players 1,3 and 4 and identifies player 2 , hence $X_{t+2}^{i}=\{2\}$ for each player $i$.

- Player 2 does not deviate in action at stage $t+1$. Then there is no action deviation at stage $t+1$ under unilateral deviations.

3. Suppose now that player 1 deviates in action and, for simplicity, does not deviate in communication at stage $t$. At stage $t+1$, players $1,2,3$ and 4 should announce respectively $\{2,3,4\},\{2,4\},\{2,3,4\}$ and $\{3,4\}$ publicly to all players. Therefore, the name of player 1 appears in at most one public announcement, whereas the names of players 2, 3 and 4 are in at least two distinct players' public announcements. Hence, $X_{t+1}^{i}=\{1,2\}$ for each player $i$.

4. Assume now that there is no action deviation at stage $t$, and that player 1 deviates in communication at stage $t+1$ by announcing $m_{t+1}^{1}(N)=N^{-1}=\{2,3,4\}$ publicly to all players: player 1 lies when he confesses his action deviation at stage $t$. At stage $t+1$, the public announcements of players 2,3 and 4 are respectively $\{1,2,3\},\{2,3,4\}$ and $\{3,4\}$, hence $X_{t}^{i}=\{1,2\}$ for each player $i$ in $N$.

5. Finally, suppose that there is no action deviation at stage $t$, and that player 1 deviates in communication at stage $t+1$ by announcing publicly $m_{t+1}^{1}(N)=N \backslash \mathcal{N}(1)=\{1,3,4\}$. The public announcements of players 2,3 and 4 at stage $t+1$ are respectively $\{1,2,3\}$, $\{2,3,4\}$ and $\{3,4\}$. All players appears in at least two messages, hence $X_{t}^{i}=\emptyset$ for each player $i$. Each player deduces that there was no action deviation at stage $t$, and that player 1 deviates in communication at stage $t+1$.

\subsubsection{Properties of the communication protocol}

I now exhibit some properties of the communication protocol constructed in the previous section. All the proofs are in the appendix. With a slight abuse of notation, I call $\tilde{\phi}^{i}$ the 
communication strategy of player $i$ which consists in sending the message $\bar{m}^{i}(N)$ when not performing the protocol, and to send messages according to the strategy prescribed by the communication protocol otherwise. The next lemma proves that, if a player who has more than two neighbors deviates in action at some stage $t$, then he is identified as suspect, and all other players are cleared at stage $t+1$.

Lemma 5.2. Consider a connected network $G$ that satisfies Condition DN, and suppose that Assumption $P$ holds. For each player $j$ such that $\sharp \mathcal{N}(j) \geq 2$, if $j$ deviates in action at some stage $t$ and if all players, except possibly a deviator, follow $\tilde{\phi}$ at stages $t$ and $t+1$, then $X_{t+1}^{i}=\{j\}$ for each player $i \in N$.

Notice that there may be a deviation from a player $d \neq j$ at stage $t+1$. Yet, in that case, player $j$ does not deviate at stage $t+1$ under unilateral deviations. The next lemma shows how the protocol works when a player $k$ has a single neighbor $\ell$.

Lemma 5.3. Consider a connected network $G$ that satisfies Condition DN, and suppose that Assumption $P$ holds. For every players $k$ and $\ell$ such that $\mathcal{N}(k)=\{\ell\}$, if:

(a) either $k$ deviates in action at stage $t$;

(b) or, there is no action deviation at stage $t, m_{t+1}^{k}=m_{t+1}^{k}(N)$ and $m_{t+1}^{k}(N) \supseteq N^{-k}$ (i.e. player $k$ confesses publicly to all players);

(c) or, there is no action deviation at stage $t, m_{t+1}^{\ell}=m_{t+1}^{\ell}(N)$ and $m_{t+1}^{\ell}(N) \supseteq N \backslash \mathcal{N}(\ell)$ (i.e. player $\ell$ claims publicly that he detected a deviation at stage $t$ );

and if all players, except possibly a deviator, follow $\tilde{\phi}$ at stages $t$ and $t+1$, then $X_{t+1}^{i}=\{k, \ell\}$ for each player $i \in N$.

The next lemma shows that no communication deviation other than those of Lemma 5.3 can induce the identification of an innocent player as a deviator.

Lemma 5.4. Consider a connected network $G$ that satisfies Condition DN, and suppose that Assumption $P$ holds. For each player $k$ in $N$, if there is no action deviation at stage $t$, and if player $k$ deviates in communication at stage $t+1$ in such a way that:

- either $\mathcal{N}(k)=\{\ell\}$, and $m_{t+1}^{k} \neq m_{t+1}^{k}(N)$ or $m_{t+1}^{k}(N) \notin\left\{N^{-k}, \mathcal{N}(k) \cup\{k\}\right\}$;

- or there exists $k^{\prime}$ such that $\mathcal{N}\left(k^{\prime}\right)=\{k\}$, and $m_{t+1}^{k} \neq m_{t+1}^{k}(N)$ or $\{N \backslash \mathcal{N}(k), \mathcal{N}(k) \cup$ $\{k\}\} \notin m_{t+1}^{k}(N)$;

- or $m_{t+1}^{k} \neq m_{t+1}^{k}(N)$ or $m_{t+1}^{k}(N) \neq\{\mathcal{N}(k) \cup\{k\}\}$

and if all players, except possibly a deviator, follow $\tilde{\phi}$ at stage $t+1$, then $X_{t+1}^{i}=\emptyset$ for each player $i \in N$. 


\subsection{The equilibrium strategy}

Take a payoff $v \in V^{*}$. In this section, I construct the PBE strategy, denoted $\left(\sigma^{*}, \phi^{*}\right)$, with payoff $v$ (the proof of the equilibrium property is in Section 5.3). More precisely, I construct a restriction of the PBE strategy to a particular class of private histories; namely, the histories along which only unilateral deviations from $\left(\sigma^{*}, \phi^{*}\right)$, if any, have taken place. Formally, I denote by $H_{t}^{i}\left(U \mid\left(\sigma^{*}, \phi^{*}\right)\right)$ the set of private histories for player $i$ such that: either no deviation (from $\left(\sigma^{*}, \phi^{*}\right)$ ) has occurred, or only unilateral deviations have taken place. That is to say, for any history in $H_{t}^{i}\left(U \mid\left(\sigma^{*}, \phi^{*}\right)\right)$, no multilateral deviation has occurred. Similarly, denote by $H_{t}\left(U \mid\left(\sigma^{*}, \phi^{*}\right)\right)$ the set of total histories along which only unilateral deviations, if any, have taken place.

I define now, for every history in $H_{t}\left(U \mid\left(\sigma^{*}, \phi^{*}\right)\right)$, a strategy profile which can be decomposed into four phases. First, there is a stream of pure action profiles that yields the desired payoff. This is how the game starts off and how it unfolds as long as no player deviates. Second, there is a communication phase (the communication protocol previously described) in case of a deviation, whose purpose is to inform the deviator's neighbors of the deviator's identity. Third, there is a punishment phase, and finally, a reward phase.

Before constructing the equilibrium strategy, notice that deviations from the equilibrium strategy are of two kinds: action and communication deviations. Since communication is costless, communication deviations have to be punished only if they affect continuation payoffs. In the strategy profile I construct, communication deviations that do not affect continuation payoffs are not punished. More precisely, if a player starts sending spurious messages although no player has deviated in action, and if in addition all players learn that there was no action deviation-i.e. all players are cleared by the communication protocol-then the deviator is not punished. On the other hand, for some communication deviations without any action deviation, it may not be possible for some players to be aware that there was no action deviation: in this case, punishments of several players take place in my construction. In any case, a player can deviate both in communication and in action, and is then punished (action deviations always lead to the deviator's punishment). Now, I construct the four phases of the strategy, then I specify the beliefs (Section 5.2.5).

\subsubsection{Phase I: equilibrium path}

For each player $i$ in $N$ and each stage $t>0$, choose $\bar{a}_{t}^{i} \in A^{i}$ such that

$$
(1-\delta) \sum_{t=1}^{\infty} \delta^{t-1} g_{t}^{i}\left(\bar{a}_{t}^{i}, \bar{a}_{t}^{\mathcal{N}(i)}\right)=v^{i}
$$


This is possible when $\delta \geq 1-\frac{1}{n}$ (existence is proved by Sorin, Proposition 4 p.151 in [32]). Moreover, Fudenberg and Maskin (1991) prove that for every $\epsilon>0$, there exists $\delta_{\epsilon}<1$ such that for all $\delta \geq \delta_{\epsilon}$ and every $v \in V^{*}$ such that $v^{i} \geq \underline{v}^{i}$ for all $i$, the deterministic sequence of pure actions $\bar{a}_{t}$ can be constructed so that the continuation payoffs at each stage are within $\epsilon$ of $v$ (Lemma 2 p. 432 in [13]). ${ }^{14}$

During this phase, player $i$ should play action $\bar{a}_{t}^{i}$ at stage $t$. Moreover, at every period, player $i$ should announce $\bar{m}_{t}^{i}(N)=\mathcal{N}(i) \cup\{i\}$ publicly to all players. This message means that player $i$ did not deviate and did not detect any action deviation at stage $t-1 .{ }^{15}$ According to player $i$, his neighbors and himself are innocent regarding any possible action deviation at stage $t-1$. Player $i$ then announces his set of innocents publicly to all players at stage $t$, denoted $I_{t}^{i}$, made of himself and his neighbors: $I_{t}^{i}=\mathcal{N}(i) \cup\{i\}$.

\subsubsection{Phase II: communication phase}

Player $i \in N$ enters phase II each time he detects a unilateral deviation from $\left(\sigma^{*}, \phi^{*}\right)$. For instance, in phase I, player $i$ enters phase II at the end of stage $t$ either when he detects an action deviation at stage $t$, or when he receives a public message different from $\bar{m}_{t}^{j}(N)=$ $\mathcal{N}(j) \cup\{j\}$ from some player $j \in N^{-i}$ at stage $t$ (only public announcements are taken into account here). During this phase, player $i$ performs the communication protocol constructed in Section 5.1.

Remark 5.5. From Lemmas 5.2, 5.3 and 5.4, it turns out that if a player $j$ deviates in action at some stage $t$, then he is identified as deviator, and can be punished. Either he is the unique suspect, or he has a single neighbor who also is suspected, in which case both can be punished. Moreover, for any pair of players $k$ and $\ell$ such that $\mathcal{N}(k)=\{\ell\}$, and if there is no action deviation at stage $t$, both $k$ and $\ell$ are identified under communication deviations (b) and (c) of Lemma 5.3; for all other communication deviations, everybody is cleared. For the latter case, all players know that there was no action deviation at stage $t$, thus only a communication deviation at stage $t+1$. That is why no player has to be punished since there is no impact on continuation payoffs. Only players $k$ 's and $\ell$ 's communication deviations that are indistinguishable with player $k$ 's action deviation have to be punished.

During phase II, players should stick to the action strategy they were playing in the previous phase. For instance, if players are following the equilibrium path at stage $t$ when they detect some deviation, they should enter the communication phase and keep playing

\footnotetext{
${ }^{14}$ If it was not the case, some player would prefer to deviate from $\bar{a}$, even if doing so caused his opponents to minmax him thereafter.

${ }^{15}$ Recall that, at each stage, messages are sent before observing stage payoffs. This assumption can be relaxed: with a slight modification, the strategy construction is still valid for the case in which messages are sent after the observation of stage payoffs.
} 
$\bar{a}_{t+1}$ at stage $t+1$. This part of the strategy is thereby purely communicative. Notice that player $i$ may start the protocol in phase I, phase III or phase IV.

I now describe how the transition is made from phase II to another phase. Denote by plan $\{j\}$ (respectively $\{k, \ell\}$ ) the punishment phase (phase III) in which player $j$ (respectively players $k$ and $\ell$ ) is minmaxed. The transition rule to another phase is the following:

- if $X_{t+1}^{i}=\emptyset$, then keep playing according to the current action phase and use the corresponding communication strategy;

- if $X_{t+1}^{i}=\{j\}$ for some player $j \in N$ such that $\sharp \mathcal{N}(j) \geq 2$ and $\sharp \mathcal{N}\left(j^{1}\right) \geq 2$ for each $j^{1} \in \mathcal{N}(j)$, then start plan $\{j\}$;

- if either $X_{t+1}^{i}=\{\ell\}$ or $X_{t+1}^{i}=\{k, \ell\}$, with $\mathcal{N}(k)=\{\ell\}$, then start plan $\{k, \ell\}$;

- otherwise, play an arbitrary Nash equilibrium of the one-shot game (history incompatible with unilateral deviations).

Remark 5.6. Assume $\mathcal{N}(k)=\{\ell\}$. Player $\ell$ may prefer plan $\{\ell\}$ to plan $\{k, \ell\}$, or the opposite. That is the reason why the transition rule prevents plan $\{\ell\}$ to happen: if player $\ell$ is identified as a suspect, then players start plan $\{k, \ell\}$ in order to minmax both, no matter whether player $k$ is also suspected. Moreover, the identification is such that player $\ell$ is always suspected when player $k$ is, so that plan $\{k\}$ never occurs either.

The next example takes up Example 5.1 to show how this transition rule works.

Example 5.7. Consider the 4-player game of Example 5.1 played on the following network:

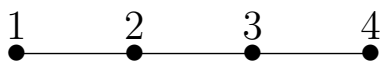

for which Condition DN is satisfied. In addition, take a payoff function $g$ for which Assumption $\mathrm{P}$ holds.

1. Assume first that player 2 deviates in action at some stage $t$ and, for simplicity, does not deviate in communication at stage $t$ (but possibly at stage $t+1$ ). From Example 5.1 , each player $i$ identifies player 2 as the deviator. The transition rule prescribes players to enter phase III in order to minmax both players 1 and 2 (plan $\{1,2\}$ ).

2. Assume now that there is no action deviation at stage $t$, and that player 2 deviates in communication at stage $t+1$ by announcing $m_{t+1}^{2}(N)=N \backslash \mathcal{N}(2)=\{2,4\}$ publicly to all players. Then, $X_{t}^{i}=\{1,2\}$ for each player $i$ in $N$. Players then start plan $\{1,2\}$. 
The reason for this joint punishment is that players 3 and 4 do not differentiate between the histories "player 1 deviates in action at stage $t$ and in communication at stage $t+1$ since he does not confess" and "player 1 deviates neither in action at stage $t$ nor in communication at stage $t+1$ and player 2 deviates in communication at stage $t+1$ ". The two following cases are then possible.

- If player 2 also deviates in action at stage $t+1$, then $X_{t+2}^{i}=\{2\}$ for each player $i$. Nevertheless, players also start plan $\{1,2\}$.

- If player 2 does not deviate in action at stage $t+1$, then there is no action deviation at stage $t+1$ under unilateral deviations. Players keep playing plan $\{1,2\}$ until a new possible deviation.

3. Assume now that player 1 deviates in action and, for simplicity, does not deviate in communication at stage $t$. Then, $X_{t+1}^{i}=\{1,2\}$ for each player $i$. Again, players 3 and 4 cannot differentiate between this deviation of player 1 and the deviation of player 2 described in the previous case. Therefore, all players start plan $\{1,2\}$.

4. Assume now that there is no action deviation at stage $t$, and that player 1 deviates in communication at stage $t+1$ by announcing $m_{t+1}^{1}(N)=N^{-1}=\{2,3,4\}$ publicly to all players: player 1 lies when he confesses his action deviation at stage $t$. Then, $X_{t}^{i}=\{1,2\}$ for each player $i$ in $N$. Again, all players start plan $\{1,2\}$.

5. Finally, assume that there is no action deviation at stage $t$, and that player 1 deviates in communication at stage $t+1$ by announcing publicly $m_{t+1}^{1}(N)=N \backslash \mathcal{N}(1)=\{1,3,4\}$. Then, $X_{t}^{i}=\emptyset$ for each player $i$ : each player deduces that there was no action deviation at stage $t$, and that player 1 deviates in communication at stage $t+1$. However, communication being costless, my construction does not require to punish player 1 . The transition rule prescribes all players to keep playing according to the phase in which the game is and to use the corresponding communication strategy.

This concludes the description of the communication phase.

\subsubsection{Phase III: punishment phase}

First case: plan $\{j\}$. Consider the situation in which each player enters a punishment phase in order to minmax player $j$. Notice first that only player $j$ 's neighbors are able to punish him. Besides, since minmax strategies might be mixed, minmaxing players' deviations 
might be undetectable: players may not know the sequences of pure actions their neighbors should play. For that reason, announcements are added in the construction. Players' strategies during phase III, denoted $\hat{\sigma}$ and $\hat{\phi}$, are as follows.

During this phase, each player's communication strategy is twofold:

(i) first, at each stage $s$, each player $i$ in $N$ announces his set of innocents $I_{s}^{i}$ publicly to all players (as in phase I). For instance, $I_{s}^{i}=\mathcal{N}(i) \cup\{i\}$ belongs to player $i$ 's public announcement at stage $s$ if player $i$ does not detect any action deviation at stage $s-1$.

(ii) In addition, each player $i$ reveals his pure action $a_{s}^{i}$ publicly to all players at each stage $s$. With these announcements, all players know the pure actions each player should have played at each stage $s$. This enables the players to detect deviations and start phase II if needed.

Recall that players choose actions and messages simultaneously at each stage, hence player $j$ has not received his opponents' announcements when he chooses his action and sends his messages. Therefore, player $j$ is indeed punished when minmax strategies are mixed.

A message of any player $i$ during phase III has then the following form: for each stage $s$ in phase III, $m_{s}^{i}(N)=\left(I_{s}^{i}, a_{s}^{i}\right)$.

Action strategies of the players are as follows. At each stage $s \geq t+2$ during phase III (the length of phase III, denoted $T(\delta)$, is adapted in Section 5.2.4):

- each player $i \in \mathcal{N}(j)$ plays according to his minmax strategy against $j$, denoted $\left(P_{j}^{i}\right)$ (recall that $P_{j}^{i}$ can be a mixed strategy). Denote by $P(j)=\left(P_{j}^{i}\right)_{i \in \mathcal{N}(j)}$ the profile of minmax strategies of player $j$ 's neighbors against him. For any strategy $\left(\sigma^{j}, \psi^{j}\right)$ of player $j$ :

$$
\begin{aligned}
\gamma_{\delta}^{j}\left(\sigma^{j}, P(j), \psi^{j},\left(\phi^{i}\right)_{i \in \mathcal{N}(j)}\right) & \leq \sum_{t=1}^{\infty}(1-\delta) \delta^{t-1} \underline{v}^{j} \\
& \leq 0
\end{aligned}
$$

where $\phi^{i}$ is an arbitrary communication strategy of a minmaxing player $i \in \mathcal{N}(j)$.

- Player $j$ commits to play an arbitrary pure action $P^{j}$ during his punishment, hence his deviations are detectable. In particular, player $j$ is supposed to play $P^{j}$ even if a player $i \in \mathcal{N}(j)$ deviates by privately reporting to player $j$ the sequence of pure actions he will play during plan $\{j\}$. Hence, player $j$ cannot use this information to get a higher 
payoff. If he deviates from $P^{j}$, then player $j$ only lengthens his punishment (see Section $5.3)$.

- The actions of other players $m \neq i, j$ are arbitrary. Recall that each player $m$ should announce publicly the pure action chosen at each stage, hence his deviations are detectable.

During phase III, each player $i$ in $N$ starts the communication phase at some stage $s$ if:

- either player $i$ detects an action deviation at stage $s-1$, i.e. his payoff at stage $s-1$ is not the one he would get if all his neighbors would have played the pure actions they announce at stage $s-1$;

- or, there exists $\tilde{i} \in \mathcal{N}(j) \cap \mathcal{N}(i)$ such that $a_{s-1}^{\tilde{i}}$ is not in the support of $P^{\tilde{i}}(k)$ (this deviation is regarded as an action deviation) ${ }^{16}$

- or, there exists a player $m \in N$ such that $I_{s}^{m} \neq \mathcal{N}(s) \cup\{s\}$.

If player $i$ never starts phase II (or if all players are cleared), then player $i$ goes to phase IV at the end of the punishment phase, hence at stage $t+2+T(\delta)$.

Second case: plan $\{k, \ell\}$. Consider now the situation in which two players $k$ and $\ell$, with $\mathcal{N}(k)=\ell$, are minmaxed from stage $t+2$ on. It means that either player $k$ or player $\ell$ have been identified as suspect at the end of stage $t+1$.

Remark 5.8. The strategy constructed in phase II is such that, whenever the state is $\{k, \ell\}$, it must be the case that $\mathcal{N}(k)=\{\ell\}$ without loss of generality (see Lemmas 5.2, 5.3 and 5.4 in Section 5.3). In this case, phase III is such that both $k$ and $\ell$ are punished. It is possible since each player has to punish only one suspect among $k$ or $\ell$.

Each player's communication strategy during phase III is the same as for the first case. However, action strategies differ and are as follows. At each stage $s \geq t+2$ during phase III:

- each player $i \in \mathcal{N}(\ell)$, including player $k$, plays according to his minmax strategy against $\ell$, denoted $\left(P^{i}(\ell)\right)$ (recall that $P^{i}(\ell)$ can be a mixed strategy).

- Player $\ell$ plays according to his minmax strategy against $k, P^{\ell}(k)$.

- The actions of other players $m \neq i, k, \ell$ are arbitrary. As explained above, each player $m$ should still announce publicly the pure action chosen at each stage.

Each player $i$ in $N$ starts phase II at some stage $s$ if:

\footnotetext{
${ }^{16}$ Notice that one can have $i=j$.
} 
- either player $i$ detects an action deviation at stage $s-1$, i.e. his payoff at stage $s-1$ is not the one he would get if all his neighbors would have played the pure action they announced at stage $s-1$;

- or there exists $\tilde{i} \in \mathcal{N}(\ell) \cap \mathcal{N}(i)$ such that $a_{s-1}^{\tilde{i}}$ is not in the support of $P^{\tilde{i}}(\ell) ;{ }^{17}$

- or $i \in \mathcal{N}(\ell)$ and $a_{s-1}^{\ell}$ is not in the support of $P^{\ell}(k)$;

- or there exists a player $m$ such that $I_{s}^{m} \neq \mathcal{N}(s) \cup\{s\}$.

If player $i$ never starts phase II (or if all players are cleared), then player $i$ goes to phase IV at the end of the punishment phase, hence at stage $t+2+T(\delta)$. This concludes the description of the punishment phase.

In the next section, I define a reward phase such that players $k$ 's and $\ell$ 's continuation payoffs after the punishment phase vary according to their realized payoff during phase III (see Section 5.2.4). Therefore, they are indifferent between the pure actions in the support of their minmax strategies. In particular, they cannot benefit from a minmaxing player reporting privately his sequence of pure actions.

\subsubsection{Phase IV: reward phase}

The aim of this phase is twofold.

(i) In order to provide each minmaxing player (who is not minmaxed himself) with an incentive to play his minmax strategy in phase III, an additional bonus $\rho>0$ is added to his average payoff. If the discount factor is large enough, the potential loss during the punishment is compensated by the future bonus.

(ii) Moreover, to induce each minmaxing player to draw his pure actions according to the right distribution of his minmax strategy, I add a phase so that his payoff in the continuation game varies with his realized payoff in a way that makes him indifferent between the pure actions in the support of his minmax strategy. As in Fudenberg and Maskin $([12,13])$, it is convenient to require that any feasible and strictly individually rational continuation payoff must be exactly attained. Otherwise, a minmaxing player might not be exactly indifferent between his pure actions in the support of his minmax strategy.

The possibility of defining such rewards relies on the full dimensionality of the payoff set (recall that int $V^{*} \neq \emptyset$ ). I use the insights of Fudenberg and Maskin (1986). The main

\footnotetext{
${ }^{17}$ Notice that one can have $i=\ell$ and $\tilde{i}=k$, or the reverse.
} 
difference here relates to the case where two players, $k$ and $\ell$, are simultaneously minmaxed during the punishment phase: the rewards are then adapted so that neither $k$ nor $\ell$ are rewarded of an additional $\rho>0$ during the reward phase (otherwise, they might have an incentive to deviate when minmaxing another player $j \neq k, \ell$ ). Moreover, all players who were not minmaxed during the punishment phase are rewarded of an additional $\rho>0$, even if they were not minmaxing during the punishment phase. The formal construction of these rewards is now given.

Choose $\left(v_{r}^{1}, \ldots, v_{r}^{n}\right) \in \operatorname{int} V^{*}$ such that for each player $i \in N, v^{i}>v_{r}^{i}$. Since $V^{*}$ has full dimension, there exists $\rho>0$ such that for each player $j$ such that $\sharp \mathcal{N}(j) \geq 2$ and $\sharp \mathcal{N}\left(j^{1}\right) \geq 2$ for every $j^{1} \in \mathcal{N}(j)$, the following holds:

$$
v_{r}(j)=\left(v_{r}^{1}+\rho, \ldots, v_{r}^{j-1}+\rho, v_{r}^{j}, v_{r}^{j+1}+\rho, \ldots, v_{r}^{n}+\rho\right) \in V^{*},
$$

and for every players $k$ and $\ell$ such that $\mathcal{N}(k)=\{\ell\}$ :

$$
v_{r}(k, \ell)=\left(v_{r}^{1}+\rho, \ldots, v_{r}^{k-1}+\rho, v_{r}^{k}, v_{r}^{k+1}+\rho, v_{r}^{\ell-1}+\rho, v_{r}^{\ell}+\frac{\rho}{2}, v_{r}^{\ell+1}+\rho, \ldots, v_{r}^{n}+\rho\right) \in V^{*} \cdot{ }^{18}
$$

For each player $i$ in $N$, let $w^{i}(d)$ be player $i$ 's realized discounted average payoff during phase III when punishing player $d$ in $N$. Denote by $\bar{g}^{i}$ player $i$ 's greatest one-shot payoff, that is:

$$
\bar{g}^{i}=\max _{a^{i},\left(a^{j}\right)_{j \in \mathcal{N}(i)}} g^{i}\left(a^{i}, a^{j}\right)
$$

Lemma 5.9. For every $\epsilon>0$ small enough, there exists $\delta^{*}>\delta_{\epsilon}{ }^{19}$ such that for all $\delta>\delta^{*}$, there exists an integer $T(\delta)^{20}$ (if several take the smallest) such that for every pair of players $i$ and $d$ :

$$
\begin{aligned}
\left(1-\delta^{2}\right) \bar{g}^{i}+\delta^{T(\delta)+3} v_{r}^{i} & <v_{r}^{i}-\epsilon \\
\left(1-\delta^{2}\right) \bar{g}^{i}+\delta^{T(\delta)+3} v_{r}^{i} & <\left(1-\delta^{T(\delta)+2}\right) w^{i}(d)+\delta^{T(\delta)+2}\left(v_{r}^{i}+\rho\right), \\
\left(1-\delta^{2}\right) \bar{g}^{i}+\delta^{T(\delta)+3} v_{r}^{i} & <\left(1-\delta^{2}\right) w^{i}(d)+\delta^{2}\left(v_{r}^{i}+\rho\right) .
\end{aligned}
$$

\footnotetext{
${ }^{18}$ This element exists since int $V^{*} \neq \emptyset$. Notice that the non-equivalent utilities condition introduced by Abreu, Dutta and Smith ([1]) is not sufficient to ensure that $v_{r}(k, \ell)$ exists and lies in $V^{*}$.

${ }^{19}$ Recall that $\delta_{\epsilon}$ is defined in Section 5.2.1 such that for every $\epsilon>0$, there exists $\delta_{\epsilon}<1$ such that for all $\delta \geq \delta_{\epsilon}$ and every $v \in V^{*}$ such that $v^{i} \geq v$ for all $i$, the deterministic sequence of pure actions $\bar{a}_{t}$ can be constructed so that the continuation payoffs at each stage are within $\epsilon$ of $v$.

${ }^{20}$ Recall that $T(\delta)$ is the length of phase III.
} 
Proof. Choose $\epsilon>0$ such that, for every players $i$ and $d, \epsilon<\min _{i} v_{r}^{i}$ and

$$
-w^{i}(d)<\frac{v_{r}^{i}-\epsilon}{v_{r}^{i}}\left(\rho-w^{i}(d)\right)
$$

This is possible since $\rho>0$. Now, I can rewrite Equation (4) as follows:

$$
0<\left(\frac{v_{r}^{i}-\epsilon}{v_{r}^{i}}\right) \rho-\left(1-\frac{v_{r}^{i}-\epsilon}{v_{r}^{i}}\right) w^{i}(d) .
$$

Then, there exists $x \in(0,1)$ such that $x v_{r}^{i}<v_{r}^{i}-\epsilon$ and $x v_{r}^{i}<(1-x) w^{i}(d)+x\left(v_{r}^{i}+\rho\right)$ (take $x$ close to but lower than $\left.\frac{v_{r}^{i}-\epsilon}{v_{r}^{i}}\right)$. Then, I choose $\delta^{*}$ close to one and $\delta^{T(\delta)}$ close to $x$ as $\epsilon$ tends to zero (notice that $x$ is close to 1 as $\epsilon$ tends to zero). The left hand side of Equations (1), (2) and (3) tends to $x v_{r}^{i}$ as $\epsilon$ tends to zero and Equations (1) and (2) directly follow. The right hand side of Equation (3) tends to $v_{r}^{i}+\frac{\rho}{2}$ as $\epsilon$ tends to zero and Equation (3) follows directly since $\rho>0$. As a consequence, $\delta^{*}$ exists.

Define now, for every pair of players $i$ and $d$ :

$$
z^{i}(d)= \begin{cases}w^{i}(d) \frac{1-\delta^{T(\delta)}}{\delta^{T(\delta)}}, & \text { if } i \in \mathcal{N}(d), \\ 0, & \text { otherwise }\end{cases}
$$

Finally, let $a_{t}\left(j, \delta,\left(z^{i}(j)\right)_{i \in \mathcal{N}(j)}\right)$ and $a_{t}\left(k, \ell, \delta,\left(z^{\ell}(k)\right),\left(z^{i}(\ell)\right)_{i \in \mathcal{N}(\ell)}\right)$ be deterministic sequences of pure actions that result in the following payoffs:

$$
\begin{gathered}
\left(v_{r}^{j},\left(v_{r}^{i}+\rho-z^{i}(j)\right)_{i \neq j}\right) \in V^{*}, \\
\left(v_{r}^{k}-z^{k}(\ell), v_{r}^{\ell}-z^{\ell}(k),\left(v_{r}^{i}+\rho-z^{i}(\ell)\right)_{i \neq k, \ell}\right) \in V^{*},
\end{gathered}
$$

and whose continuation payoffs are within $\epsilon$ of (5) and (6) respectively.

Lemma 5.10. The sequences $a_{t}\left(j, \delta,\left(z^{i}(j)\right)_{i \in \mathcal{N}(j)}\right)$ and $a_{t}\left(k, \ell, \delta,\left(z^{\ell}(k)\right),\left(z^{i}(\ell)\right)_{i \in \mathcal{N}(\ell)}\right)$ exist for $\epsilon$ close to zero and $\delta$ close to one.

Proof. Consider a sequence $\left(\epsilon_{n}, \delta_{n}\right)$ such that $\epsilon_{n}$ tends to zero and $\delta_{n}$ tends to one as $n$ tends to infinity. By construction, $T(\delta)$ is the smallest integer satisfying equations (3), (4) and $(5)$, and $\delta^{T(\delta)}$ is close to $\frac{v_{r}^{i}-\epsilon}{v_{r}^{i}}$. Hence, $\delta_{n}^{\mu\left(\delta_{n}\right)}$ is close to one for $n$ sufficiently large, which implies that $z^{i}(j)=w^{i}(j) \frac{1-\delta_{n}^{\mu\left(\delta_{n}\right)}}{\delta_{n}^{\mu\left(\delta_{n}\right)}}$ tends to zero as $n$ tends to infinity. As a consequence, for $n$ sufficiently large, $\rho-z^{i}(j)>0$ and the payoffs in (8) are in $V^{*}$ and bounded away from the axes by at least $\epsilon_{n}$. By Lemma 2 in Fudenberg and Maskin (page 432 in [13]), this implies that for $n$ sufficiently large, there exists $\bar{\delta}$ close to one (and greater than $\delta^{*}>\delta_{\epsilon}$ ) such that, 
for every $\delta>\bar{\delta}$, there exists a sequence of pure actions $a_{t}\left(j, \delta_{n},\left(z^{i}(j)\right)_{i \in N}\right)$ with payoffs $(8)$ and whose continuation payoffs are within $\epsilon_{n}$ of (8). Similar arguments apply to prove that $a_{t}\left(k, \ell, \delta,\left(z^{\ell}(k)\right),\left(z^{i}(\ell)\right)_{i \in \mathcal{N}(\ell)}\right)$ exists.

The strategy $\sigma^{i *}$ for any player $i$ in phase IV is then the following. On one hand, if only player $j$ is minmaxed in phase III, player $i$ starts playing $a_{t}\left(j, \delta,\left(z^{i}(j)\right)_{i \in N}\right)$ at stage $t+3+T(\delta)$ until a new possible deviation. On the other hand, if both players $k$ and $\ell$ are minmaxed in phase III, then player $i$ starts playing $a_{t}\left(k, \ell, \delta,\left(z^{\ell}(k)\right)_{i \in \mathcal{N}(k)},\left(z^{i}(\ell)\right)_{i \in \mathcal{N}(\ell)}\right)$ at stage $t+3+T(\delta)$ until a new possible deviation.

Intuitively, these rewards are such that, if plan $\{j\}$ or plan $\{k, \ell\}$, with $\mathcal{N}(k)=\{\ell\}$, is played, each player $i \neq k, \ell$ has an incentive to play his minmax strategy against $j$ due to the additional bonus of $\rho$ thereafter. In addition, players $k$ and $\ell$ have no incentive to deviate during plan $\{k, \ell\}$, otherwise they would only lengthen their punishment, postponing positive payoffs (recall that by construction plans $\{k\}$ and $\{\ell\}$ are never played). Finally, when punishing any player $d$ in $N$, each minmaxing player $i$ has no incentive to draw the sequence of pure actions according to another distribution, whose support is included in the support of $P^{i}(d)$ : any expected advantage that player $i$ gets from playing some pure action in phase III is subsequently removed in phase IV.

For each player $i \in N, \phi^{*}$ is the same as before: when a player $i$ does not detect any deviation at stage $t$, then he should send the message $m_{t+1}^{i}=\mathcal{N}(i) \cup\{i\}$. When a player $i$ detects a deviation, he starts phase II.

Finally, notice that play after histories that involve simultaneous deviations is irrelevant. Hence, the partial strategy that I define can be completed in any arbitrary fashion. This concludes the construction of $\left(\sigma^{*}, \phi^{*}\right)$. The next section specifies the beliefs.

\subsubsection{Specification of the beliefs}

A belief assessment is a sequence $\mu=\left(\mu_{t}^{i}\right)_{t \geq 1, i \in N}$ with $\mu_{t}^{i}: H_{t}^{i} \rightarrow \Delta\left(H_{t}\right)$ : given a private history $h^{i}$ of player $i, \mu_{t}^{i}\left(h^{i}\right)$ is the probability distribution representing the belief that player $i$ holds on the full history. An assessment is an element $((\sigma, \phi), \mu)$ where $(\sigma, \phi)$ is a strategy profile and $\mu$ a belief assessment.

I consider a restricted set of beliefs, which is strictly included in the set of total histories $H_{t}$. I call this set of beliefs $\mathcal{B}=\left(\mathcal{B}^{i}\right)_{i \in N}$. Namely, for each player $i$ in $N$, every belief in $\mathcal{B}^{i}$ only assigns positive probability to histories that differ from equilibrium play, $\left(\sigma^{*}, \phi^{*}\right)$, in as much as, and to the minimal extent which, their private history dictates that it does. Formally, for every player $i$ in $N$ and every history $h_{t}^{i} \in H_{t}^{i}$, I denote by $H_{t}\left[h_{t}^{i}\right] \subset H_{t}$ the set

of total histories for which the projection on $H_{t}^{i}$ is $h_{t}^{i}$. A total history $h_{t}$ in $H_{t}\left[h_{t}^{i}\right]$ is said to 
be compatible with private history $h_{t}^{i}$ of player $i$. Now, for every player $i$ in $N$ and every history $h_{t}^{i} \in H_{t}^{i}$, let $H_{t}\left[h_{t}^{i}\right]\left(U \mid\left(\sigma^{*}, \phi^{*}\right)\right) \subseteq H_{t}\left[h_{t}^{i}\right]$ be the set containing all the total histories that are compatible with $h_{t}^{i}$ and included in $H_{t}\left(U \mid\left(\sigma^{*}, \phi^{*}\right)\right) .{ }^{21}$ Formally, for each player $i$ in $N$ and every history $h_{t}^{i} \in H_{t}^{i}$ :

$$
H_{t}\left[h_{t}^{i}\right]\left(U \mid\left(\sigma^{*}, \phi^{*}\right)\right)=H_{t}\left[h_{t}^{i}\right] \cap H_{t}\left(U \mid\left(\sigma^{*}, \phi^{*}\right)\right) .
$$

The set of beliefs $\mathcal{B}^{i}$ is then the following:

$\mathcal{B}^{i}=\left\{\left(\mu_{t}^{i}\right)_{t \geq 1}: \forall t \geq 1, \forall h_{t}^{i} \in H_{t}^{i}, h_{t}^{i} \in H_{t}^{i}\left(U \mid\left(\sigma^{*}, \phi^{*}\right)\right) \Rightarrow \operatorname{supp} \mu_{t}^{i}\left(h_{t}^{i}\right) \subseteq H_{t}\left[h_{t}^{i}\right]\left(U \mid\left(\sigma^{*}, \phi^{*}\right)\right)\right\}$,

where supp stands for the support of $\mu_{t}^{i}\left(h_{t}^{i}\right)$. In other words, the beliefs of the players are such that, if they observe a history compatible with either no deviation or unilateral deviations, then they assign probability one to the fact that the total history is in $H_{t}\left(U \mid\left(\sigma^{*}, \phi^{*}\right)\right)$ and is compatible with $h_{t}^{i}$.

In the next section, I show that for every $\mu \in \mathcal{B},\left(\left(\sigma^{*}, \phi^{*}\right), \mu\right)$ is a PBE with payoff $v^{*}$.

\subsection{The equilibrium property}

I now prove the following proposition, which implies the sufficiency part of Theorem 4.1.

Proposition 5.11. Assume that $G$ is such Condition DN holds, that $g$ satisfies Assumption $P$, and that int $V^{*} \neq \emptyset$. Then, for every $v \in V^{*}$, there exists $\bar{\delta} \in(0,1)$ such that for all $\delta \in(\bar{\delta}, 1)$, the assessment $\left(\left(\sigma^{*}, \phi^{*}\right), \mu\right)$, for each $\mu \in \mathcal{B}$, is a PBE with payoff $v$ in the $\delta$-discounted game.

Proof. Assume that $G$ is such that Condition DN holds, that $g$ satisfies Assumption $\mathrm{P}$, and that int $V^{*} \neq \emptyset$. Take $v \in V^{*}, \bar{\delta}$ (defined in Section 5.2.4), and consider $\delta>\bar{\delta}$. Recall that $\bar{\delta}>\delta_{\epsilon}$ so that the sequence of pure actions $\bar{a}_{t}$ defined in Section 5.2.1 exists and the continuation payoffs are within $\epsilon$ of $v$.

Since, play after histories that involve multilateral deviations is irrelevant, consider the strategy $\left(\sigma^{*}, \phi^{*}\right)$ constructed in the previous sections for all private histories in $H_{t}^{i}\left(U \mid\left(\sigma^{*}, \phi^{*}\right)\right)$, for each player $i$ in $N$. Take a belief assessment $\mu$ in $\mathcal{B}$. I now prove that $\left(\left(\sigma^{*}, \phi^{*}\right), \mu\right)$ is a PBE.

Take first a player $j$ such that $\sharp \mathcal{N}(j) \geq 2$ and $\sharp \mathcal{N}(i) \geq 2$ for each $i \in \mathcal{N}(j)$. By Lemma 5.4 , player $j$ 's communication deviations do not change continuation strategies, provided player

\footnotetext{
${ }^{21}$ Recall that $H_{t}\left(U \mid\left(\sigma^{*}, \phi^{*}\right)\right)$ is the set of total histories along which only unilateral deviations, if any, have taken place.
} 
$j$ does not deviate in action (recall that the deviation which consists in falsely reporting his pure actions during phase III is regarded as an action deviation). Henceforth, I focus on player $j$ 's action deviations. Assume that player $j$ stops playing action $\bar{a}_{t}^{j}$ at some stage $t$ during phase I and then conforms; without loss of generality, let $t=1$. Lemmas 5.2 and 5.3 imply that player $j$ is identified as the deviator at stage $t+1=2$ by all players and the state becomes $\{j\}$. Player $j$ is thus minmaxed at stage $t+2=3$ during $T(\delta)$ periods. Player $j$ 's discounted expected payoff is then no more than:

$$
(1-\delta) \sum_{t=1}^{2} \delta^{t-1} \bar{g}^{j}+(1-\delta) \sum_{t>T(\delta)+3} \delta^{t-1} v_{r}^{j}=\left(1-\delta^{2}\right) \bar{g}^{j}+\delta^{T(\delta)+3} v_{r}^{j} .
$$

Since $\delta>\bar{\delta}>\delta^{*}$, Equation (1) ensures that this is less than $v_{r}^{j}-\epsilon$ which is a lower bound for player $j$ 's continuation payoff for conforming from date $t$ on.

If player $j$ deviates in action during phase III when he is being punished, he obtains at most zero the stage in which he deviates, and then only lengthens his punishment, postponing the positive payoff $v_{r}^{j}$. The case where player $j$ deviates in action in phase III when a player $d \notin \mathcal{N}(j)$ is being punished is also trivial, since by construction player $j$ 's action is arbitrary and may be a best-response.

Assume now that player $j$ deviates in action at stage $t$ during phase III when player $d \in \mathcal{N}(j)$ is being punished and then conforms. Two cases are possible. Assume first that player $j$ deviates at stage $t$ by playing an action which is not in the support of his minmax strategy. By construction, player $j$ is identified as deviator at stage $t+1$. Player $j$ 's discounted expected payoff from the beginning of stage $t$ is thus no more than:

$$
(1-\delta) \sum_{t=1}^{2} \delta^{t-1} \bar{g}^{j}+(1-\delta) \sum_{t>T(\delta)+3} \delta^{t-1} v_{r}^{j}=\left(1-\delta^{2}\right) \bar{g}^{j}+\delta^{T(\delta)+3} v_{r}^{j} .
$$

On the contrary, if he conforms, he gets at least:

$$
\left(1-\delta^{T(\delta)-t+2}\right) w^{j}(d)+\delta^{T(\delta)-t+2}\left(v_{r}^{j}+\rho\right) .
$$

If $t=1$, then Equation (2) implies that Equation (8) exceeds Equation (7). If $t=T(\delta)$, it follows from Equation (3) that Equation (8) exceeds Equation (7). Finally, the cases for which $1<t<T(\delta)$ follow from Equations (2) and (3) combined.

Second, Phase IV is constructed so that player $j$ is indifferent among all actions in the support of his minmax strategy during phase III when player $i$ is punished (if player $j$ conforms during phase IV). Regardless of player $j$ 's actions in this phase, his continuation 
payoff from the beginning of phase III is within $\epsilon$ of:

$$
(1-\delta) \sum_{t=1}^{T(\delta)} \delta^{t-1} g^{j}\left(a_{t}^{j}, a_{t}^{\mathcal{N}(j)}\right)+\delta^{T(\delta)}\left(v_{r}^{j}+\rho-z^{j}(i)\right)=\delta^{T(\delta)}\left(v_{r}^{j}+\rho\right)
$$

Hence, player $j$ has no incentive to deviate in phase III by randomizing according to another distribution, whose support is included in the support of his minmax strategy.

Finally, if player $j$ deviates in action at stage $t$ during phase IV, his discounted expected payoff is no more than:

$$
(1-\delta) \sum_{t=1}^{2} \delta^{t-1} \bar{g}^{j}+(1-\delta) \sum_{t>T(\delta)+3} \delta^{t-1} v_{r}^{j}=\left(1-\delta^{2}\right) \bar{g}^{j}+\delta^{T(\delta)+3} v_{r}^{j}
$$

If player $j$ conforms, his continuation payoff is at least $v_{r}^{j}-\epsilon$, and so Equation (1) ensures that deviation is not profitable.

Take now a pair of players $k$ and $\ell$ such that $\mathcal{N}(k)=\ell$. If player $k$ (respectively player $\ell$ ) deviates only in communication, then either continuation strategies do not change (in the case in which all players are cleared, see Lemma 5.4), or both players $k$ and $\ell$ are minmaxed (see Lemma 5.3) and similar arguments as for action deviations apply. Moreover, similar arguments as before (cases in which player $j$ deviates) show that neither player $k$ nor player $\ell$ has an incentive to deviate in action during any phase of the game. (Notice that even if neither player $k$ nor $\ell$ obtain a reward after plan $\{k, \ell\}$ although they are minmaxing players, they still have an incentive to play their minmax strategies, otherwise they would lengthen their punishment, postponing positive payoffs.)

To conclude, notice that the proof of the optimality of $\left(\sigma^{*}, \phi^{*}\right)$ above does not take into account the beliefs. Indeed, since $\mu^{i}$ in $\mathcal{B}^{i}$, for any history in $H_{t}^{i}\left(U \mid\left(\sigma^{*}, \phi^{*}\right)\right)$, each player $i$ in $N$ believes that there is either no deviation or only unilateral deviations. The partial strategy $\left(\sigma^{*}, \phi^{*}\right)$ prevents player $i$ from deviating, no matter what his beliefs (in $\mathcal{B}^{i}$ ) are. Indeed, under $\mu^{i}$, player $i$ believes that if he deviates, it will lead either to his punishment, or to no changes in his continuation payoff (in case of a communication deviate only).

Remark 5.12. One can deduce from the previous proof that the strategy $\left(\left(\sigma^{*}, \phi^{*}\right), \mu\right)$ is belieffree for each history in $H_{t}\left(U \mid\left(\sigma^{*}, \phi^{*}\right)\right)$ and for each belief assessment in $\mu \in \mathcal{B}$. Formally, I denote by $\left(\left(\sigma^{i}, \phi^{i}\right), \mu^{i}\right) \mid h_{t}^{i}$ player $i$ 's continuation assessment after private history $h_{t}^{i}$. Let also $\left(\left(\sigma^{-i}, \phi^{-i}\right), \mu^{-i}\right) \mid h_{t}^{-i}$ be the profile of continuation assessments of players $j \neq i$ after private histories $h_{t}^{j}$. The assessment $\left(\left(\sigma^{*}, \phi^{*}\right), \mu\right)$ satisfies the following: for every $h_{t} \in H_{t}\left(U \mid\left(\sigma^{*}, \phi^{*}\right)\right)$, 
every $\mu \in \mathcal{B}$ and every $i \in N$ :

$$
\left(\left(\sigma^{i}, \phi^{i}\right), \mu^{i}\right) \mid h_{t}^{i} \in \mathrm{BR}\left(\left(\left(\sigma^{-i}, \phi^{-i}\right), \mu^{-i}\right) \mid h_{t}^{-i}\right)
$$

where BR stands for the best-reply function of player $i$. In other words, $\left(\left(\sigma^{*}, \phi^{*}\right), \mu\right)$ is such that, for every player $i$, every private history $h_{t}^{i} \in H_{t}^{i}\left(U \mid\left(\sigma^{*}, \phi^{*}\right)\right)$, every $\mu^{i} \in \mathcal{B}^{i}$ :

$$
\left(\left(\sigma^{i}, \phi^{i}\right), \mu^{i}\right) \mid h_{t}^{i} \in \mathrm{BR}\left(\left(\left(\sigma^{-i}, \phi^{-i}\right), \mu^{-i}\right) \mid h_{t}^{-i}\right),
$$

for every $\mu^{-i} \in \mathcal{B}^{-i}$ and every $h_{t}^{-i} \in H_{t}^{-i}(U)$ that are possible given the monitoring structure.

\section{Necessity of Condition DN}

Condition DN of Theorem 4.1 is necessary for the folk theorem to hold.

Proposition 6.1. Assume that $G$ does not satisfy Condition DN. Then, there exists a payoff function $g$ such that: Assumption $P$ holds, int $V^{*} \neq \emptyset$, and there exists a feasible and strictly individually rational payoff $v \in V^{*}$ such that $v$ is not a Nash equilibrium payoff of the repeated game.

The proof builds on the arguments developed in [23] (Section 3 page 720), and is thus relegated to Appendix A. Intuitively, when Condition DN is violated, there exists a player $i$, two of his neighbors $j$ and $k$, and two deviations of $j$ and $k$ such that: for any action profile (possibly mixed), both $j$ 's and $k$ 's deviations induce the same distribution over player $i$ 's payoffs, and the same distribution over the messages received by player $i .^{22}$ The payoffs are constructed so that there exists a feasible and strictly individually rational payoff which is not jointly rational (in the sense of Renault and Tomala in [30]; see Example 3.1 therein for a similar phenomenon).

\section{Concluding remarks}

In this section, I introduce some extensions of the model, and state some open problems.

Strict incentives to confess. Part of the equilibrium strategy constructed in Section 5 is weakly dominated. Indeed, a player who deviates at some stage $t$ is required to confess at stage $t+1$. Nevertheless, it is possible to make the players have strict incentives to confess,

\footnotetext{
${ }^{22}$ Following the terminology of Fudenberg, Levine and Maskin ([11]), pairwise identifiability fails.
} 
by adjusting accordingly the reward in phase IV: indeed, the reward phase can be such that a player who deviates, and confesses publicly his deviation after that, obtains a bonus of $\frac{\rho}{2}>0$ during phase IV. Hence, a player who deviates has a strict incentive to confess. In addition, this bonus is strictly lower than $\rho$, otherwise a minmaxing player might have an incentive to deviate. Indeed, a minmaxing player's realized payoff during phase III may be less than if he would get his minmax level instead. However, a minmaxing player has still no incentive to deviate, otherwise he would loose $\frac{\rho}{2}$ at each stage during phase IV.

Correlated minmax. In some repeated games with imperfect monitoring, it is possible to drive equilibrium payoffs below the independent minmax, see Renault and Tomala ([29]) for illuminating examples. It is the case here: Theorem 4.1 remains unchanged if I rather consider correlated minmax, defined as follows:

$$
\bar{w}^{i}=\min _{x^{\mathcal{N}(i)} \in \Delta\left(A^{\mathcal{N}}(i)\right.} \max _{x^{i} \in \Delta\left(A^{i}\right)} g^{i}\left(x^{i}, x^{\mathcal{N}(i)}\right) .
$$

To prove Theorem 4.1 in that case, I adapt the strategy constructed in Section 5 in the following way. The idea is that players can correlate their actions when punishing player $k$ (respectively players $k$ and $\ell$ ) in phase III, without revealing information to the minmaxed player(s). For that, define $Q^{N(k)}(k) \in \Delta\left(A^{\mathcal{N}(k)}\right)$ (respectively $Q^{N(\ell)}(\ell) \in \Delta\left(A^{\mathcal{N}(\ell)}\right)$ ) a correlated strategy that realizes the minimum in $\bar{w}^{k}$ (respectively $\bar{w}^{\ell}$ ). Choose a player $j \neq k$ (respectively $j \neq k, \ell$ ). At the beginning of phase III, I add a stage in which player $j$ draws i.i.d. sequences of pure actions according to $Q^{N(k)}(k)$ (respectively $Q^{N(\ell)}(\ell)$ when both players $k$ and $\ell$ are minmaxed $)^{23}$ for the minmaxing players for $T(\delta)$ periods. Player $j$ announces the sequences publicly to all players except $k$ (respectively player $\ell$ ). Deviations of player $j$ are punished as before, and the reward phase makes player $j$ indifferent between the pure actions actually played by him and his neighbors (recall that the reward phase is based on player $j$ 's realized payoff).

Private communication An alternative model would be to consider private announcements, i.e. the list of receivers of a message is not certifiable. The construction requires the possibility for the players to make public announcements in two cases only.

(i) First, if there exists a player $k$ such that $\mathcal{N}(k)=\{\ell\}$, then players $k$ and $\ell$ have to make public announcements in phase II of the construction. Otherwise, a communication deviation of player $k$ (respectively player $\ell$ ) could be to send spurious messages

\footnotetext{
${ }^{23}$ Notice that if $\mathcal{N}(k)=\{\ell\}$, and both players $k$ and $\ell$ are minmaxed, there is no need to correlate in order to punish player $k$ since only player $\ell$ is a neighbor of $k$.
} 
to a subset of players only. With the possibility of public communication, the strategy constructed in Section 5 ignores such deviations. If public communication is not allowed, this implies a lack of common knowledge of the deviation's date, and there may be a coordination failure with some players starting phase III whereas other do not.

(ii) Second, public announcements are crucial in the punishment phase (phase III). Otherwise, some communication deviations may entail a coordination failure, since players could have different informations on the pure actions chosen by their opponents (recall that pure actions are announced in phase III).

Otherwise, only private communication is required. Hence, the following corollary holds:

Corollary 7.1. Assume that players are only allowed to communicate privately with each other (no certifiability). If Condition DN holds, and if each player has more than two neighbors, then the folk theorem holds with minmax levels in pure strategies (and PBE as solution concept).

The proof is a straightforward application of the proof of Theorem 4.1.

Folk theorem without discounting. Condition DN of Theorem 4.1 is also the necessary and sufficient condition for a folk theorem to hold if I consider uniform (sequential) equilibria of the undiscounted repeated game (see [10] and [33]). Namely, every feasible and individually rational payoff is a uniform (sequential) equilibrium payoff for any payoff function $g$ that satisfies Assumption P if and only if Condition DN holds. Moreover, in that case, public announcements are not required, and players can be only allowed to send private messages (coordination in cases (i) and (ii) above is not required). In addition, it is possible to restrict communication along the network: the necessary and sufficient conditions for a folk theorem to hold are known (but are different from Condition DN) if (i) only private communication is allowed (no public announcements) and (ii) I consider Nash equilibrium of the repeated game or uniform sequential equilibrium for the undiscounted case ([23]). If communication is restricted along the network, finding conditions for a folk theorem to hold is an open problem for (i) public announcements and (ii) sequential equilibria of repeated games with discounting. 


\section{Appendix}

\section{A Proof of Proposition 6.1}

\section{A.1 Proof}

Take a network $G$ such that Condition DN does not hold. It implies that there exists two players $j$ and $k$ in $N$, who have the same neighbors: $\mathcal{N}(j) \backslash\{k\}=\mathcal{N}(k) \backslash\{j\}$. For brevity, I focus on the case where players $j$ and $k$ are not neighbors. The proof can be easily extended to the case where $j$ and $k$ are neighbors.

Take a player $i \in \mathcal{N}(j)$. Notice that all other players, $m \neq i, j, k$ are either neighbors of both $j$ and $k$, or of none of them. Consider the payoff function for players $i, j$ and $k$ represented by the following table (where player $i$ chooses the row, player $j$ the column, and player $k$ the matrix): ${ }^{24}$

\section{$k$ plays $C$}

\begin{tabular}{|c|c|c|}
\hline$i \backslash j$ & $C$ & $D$ \\
\hline$C$ & $1,0,2$ & $0,0,2$ \\
\hline$D$ & $1,2,0$ & $0,6,0$ \\
\hline
\end{tabular}

$k$ plays $D$

\begin{tabular}{|c|c|c|}
\hline$i \backslash j$ & $C$ & $D$ \\
\hline$C$ & $0,0,6$ & $1,0,6$ \\
\hline$D$ & $0,2,0$ & $1,6,0$ \\
\hline
\end{tabular}

I write $u\left(a^{i}, a^{j}, a^{k}\right)$ for this payoff vector. Player $j$ 's payoff does not depend on $k$ 's action, nor does $k$ 's payoff depends on $j$ 's action. Accordingly, I write $u^{j}\left(a^{j}, a^{i}\right)$ and $u^{k}\left(a^{k}, a^{i}\right)$ in what follows. To complete the description of $g$, each player $m \neq i, j, k$ has two actions $C$ and $D$ such that:

- for each player $m$ such that $m \notin \mathcal{N}(j) \cap \mathcal{N}(k)$, player $m$ 's payoff at stage $t$ is:

$$
g^{m}\left(a_{t}^{m}, a_{t}^{\mathcal{N}(m)}\right)=\ell_{t} \frac{\epsilon}{n}
$$

for some $\epsilon>0$, and $\ell_{t}=\sharp\left\{\ell: \ell \in \mathcal{N}(m) \cup\{m\}\right.$ and $\left.a_{t}^{\ell}=C\right\}\left(\ell_{t}\right.$ is the number of $m$ 's neighbors including himself who play $C$ at stage $t$ );

- for each player $m$ such that $m \in \mathcal{N}(j) \cap \mathcal{N}(k)$, player $m$ 's payoff at stage $t$ is:

$$
g^{m}\left(a_{t}^{m}, a_{t}^{\mathcal{N}(m)}\right)= \begin{cases}1+\ell_{t}^{-j k} \frac{\epsilon}{n} & \text { if } a^{j}=a^{k} \\ \ell_{t}^{-j k} \frac{\epsilon}{n} & \text { otherwise }\end{cases}
$$

\footnotetext{
${ }^{24}$ In the case where $j$ and $k$ are neighbors, I assume that if player $j$ plays $D$ it adds $\epsilon>0$ to player $k$ 's payoff, and symmetrically if player $k$ plays $D$ it adds $\epsilon>0$ to player $j$ 's payoff. This ensures that Assumption $\mathrm{P}$ holds.
} 
for some $\epsilon>0$, and $\ell_{t}^{-j k}=\sharp\left\{\ell: \ell \in \mathcal{N}(m) \backslash\{j, k\} \cup\{m\}\right.$ and $\left.a_{t}^{\ell}=C\right\}\left(\ell_{t}\right.$ is the number of $m$ 's neighbors distinct from $j$ and $k$, including himself, who play $C$ at stage $t)$

- for players $i, j$ and $k$ :

$$
\begin{aligned}
g^{i}\left(a_{t}^{i}, a_{t}^{\mathcal{N}(i)}\right) & =u^{i}\left(a_{t}^{i}, a_{t}^{j}, a_{t}^{k}\right)-\ell_{t}^{i} \frac{\epsilon}{n}, \\
g^{j}\left(a_{t}^{j}, a_{t}^{\mathcal{N}(j)}\right) & =u^{j}\left(a_{t}^{j}, a_{t}^{i}\right)+\ell_{t}^{j} \frac{\epsilon}{n}, \\
g^{k}\left(a_{t}^{k}, a_{t}^{\mathcal{N}(k)}\right) & =u^{k}\left(a_{t}^{k}, a_{t}^{i}\right)+\ell_{t}^{k} \frac{\epsilon}{n},
\end{aligned}
$$

where $\ell_{t}^{i}=\sharp\left\{\ell: \ell \in \mathcal{N}(i) \backslash\{j, k\}\right.$ and $\left.a_{t}^{\ell}=D\right\}, \ell_{t}^{j}=\sharp\left\{\ell: \ell \in \mathcal{N}(j) \backslash\{i\}\right.$ and $\left.a_{t}^{\ell}=D\right\}$, $\ell_{t}^{k}=\sharp\left\{\ell: \ell \in \mathcal{N}(k) \backslash\{i\}\right.$ and $\left.a_{t}^{\ell}=D\right\}$ and $u^{i}\left(a_{t}^{i}, a_{t}^{j}, a_{t}^{k}\right), u^{j}\left(a_{t}^{j}, a_{t}^{i}\right)$, and $u^{k}\left(a_{t}^{k}, a_{t}^{i}\right)$ are defined by the matrix above.

This payoff function $g$ has the following properties: ${ }^{25}$

(i) $g$ satisfies Assumption P;

(ii) $\operatorname{int} V^{*} \neq \emptyset$;

(iii) $\underline{v}^{i}<0, \underline{v}^{j}=0$, and $\underline{v}^{k}=0$;

(iv) $C$ is a dominant strategy for each player $\ell \neq i, j, k$;

(v) the outcome $(1,1,1)$ (representing the payoffs of players $i, j$ and $k$ ) is in $V^{*}$;

(vi) if $a^{\ell} \neq C$ for every $\ell \in \mathcal{N}(i)$, then $g^{i}\left(a_{t}^{i}, a_{t}^{\mathcal{N}(i)}\right)<1$. Hence, the unique way to obtain the outcome $(1,1,1)$ is that player $i$ chooses between $C$ and $D$ with probability $\frac{1}{2}-\frac{1}{2}$, and that all his neighbors (including players $j$ and $k$ ) take action $C$;

(vii) player $i$ cannot punish both players $j$ and $k$ : player $i$ has to play $C$ in order to minmax player $j$, which yields a payoff of 6 for player $k$; and player $i$ has to choose action $D$ in order to minmax player $k$, which yields a payoff of 6 for player $j$;

(viii) for each player $m \in \mathcal{N}(j) \cap \mathcal{N}(k)$ (including player $i$ ), for every $a^{m} \in\{C, D\}$, and for every $a^{\mathcal{N}(m) \backslash\{j, k\}}$, the following properties hold:

$$
\begin{aligned}
& g^{m}\left(a^{m}, a^{\mathcal{N}(m) \backslash\{j, k\}}, a^{j}=C, a^{k}=C\right)=g^{m}\left(a^{m}, a^{\mathcal{N}(m) \backslash\{j, k\}}, a^{j}=D, a^{k}=D\right), \\
& g^{m}\left(a^{m}, a^{\mathcal{N}(m) \backslash\{j, k\}}, a^{j}=C, a^{k}=D\right)=g^{m}\left(a^{m}, a^{\mathcal{N}(m) \backslash\{j, k\}}, a^{j}=D, a^{k}=C\right) .
\end{aligned}
$$

\footnotetext{
${ }^{25}$ It is possible to construct a payoff function $g$ which satisfies these properties when players have more than two actions. This construction is described in the next section (A.2).
} 
Assume now that $(1,1,1)$ is a Nash equilibrium of the repeated game, and let the profiles $\bar{\sigma}=\left(\bar{\sigma}^{i}, \bar{\sigma}^{j}, \bar{\sigma}^{k},\left(\bar{\sigma}^{m}\right)_{m \neq i, j, k}\right)$ and $\bar{\phi}=\left(\bar{\phi}^{i}, \bar{\phi}^{j}, \bar{\phi}^{k},\left(\bar{\phi}^{m}\right)_{m \neq i, j, k}\right)$ be an equilibrium yielding a payoff of $\gamma_{\delta}=(1,1,1)$ for players $i, j$ and $k$. I construct deviations $\left(\tau^{j}, \psi^{j}\right)$ and $\left(\tau^{k}, \psi^{k}\right)$ for players $j$ and $k$ such that:

(1) both deviations induce the same probability distributions over the sequences of messages and payoffs received by player $i$ (deviations are indistinguishable).

(2) I will deduce from (1) that: $\gamma^{j}\left(\tau^{j}, \bar{\sigma}^{-j}, \psi^{j}, \bar{\phi}^{-j}\right)+\gamma^{k}\left(\tau^{k}, \bar{\sigma}^{-k}, \psi^{k}, \bar{\phi}^{-k}\right) \geq 3$.

The latter equation contradicts that $(\bar{\sigma}, \bar{\phi})$ is an equilibrium of the repeated game. Indeed, $\gamma^{j}(\bar{\sigma}, \bar{\phi})+\gamma^{k}(\bar{\sigma}, \bar{\phi})=2$ and $\gamma^{j}\left(\tau^{j}, \bar{\sigma}^{-j}, \psi^{j}, \bar{\phi}^{-j}\right)+\gamma^{k}\left(\tau^{k}, \bar{\sigma}^{-k}, \psi^{k}, \bar{\phi}^{-k}\right) \geq 3$. Therefore, either $\left(\tau^{j}, \psi^{j}\right)$ is a profitable deviation for player $j$, or $\left(\tau^{k}, \sigma^{k}\right)$ is a profitable deviation for player $k$. I now construct these deviations. Define $\left(\tau^{j}, \psi^{j}\right)$ as follows (the construction of $\left(\tau^{k}, \psi^{k}\right)$ is symmetric):

- at each stage, player $j$ chooses between $C$ and $D$ with probability $\frac{1}{2}-\frac{1}{2}$ (instead of $C$ with probability 1);

- player $j$ uses the communication strategy $\psi^{j}=\bar{\phi}^{j}\left(h^{j}\left(\tau^{k}, \sigma^{-k}, \psi^{k}, \phi^{-k}\right)\right)$, where the element $h^{j}\left(\tau^{k}, \sigma^{-k}, \psi^{k}, \phi^{-k}\right)$ stands for an arbitrary private history of player $j$ in which player $k$ plays according to $\left(\tau^{k}, \psi^{k}\right)$, and all other players follow $\left(\sigma^{-k}, \phi^{-k}\right)$ : player $j$ follows the equilibrium communication strategy but replaces the true history by the fictitious one in which player $k$ is the deviator.

Under such a deviation, player $i$ has no way to deduce whether $j$ or $k$ deviated, even when $(\bar{\sigma}, \bar{\phi})$ is a mixed strategy. Indeed, for every pure action profile, both player $j$ 's and $k$ 's deviations induce the same payoffs for player $i$, since $g$ satisfies property (viii). In addition, notice that player $i$ 's payoff is 1 if $j$ and $k$ choose the same action, and 0 otherwise. Therefore, since $\tau^{j}$ and $\tau^{k}$ prescribe to choose $C$ and $D$ with probability $\frac{1}{2}-\frac{1}{2}$ at each stage, $\left(\tau^{j}, \psi^{j}, \bar{\sigma}^{-j}, \bar{\phi}^{-j}\right)$ and $\left(\tau^{k}, \psi^{k}, \bar{\sigma}^{-k}, \bar{\phi}^{-k}\right)$ induce the same distribution over the payoffs of player $i$, even if $\bar{\sigma}^{j}$ and $\bar{\sigma}^{k}$ are mixed: player $i$ gets a payoff of 1 or 0 with probability $\frac{1}{2}-\frac{1}{2} \cdot{ }^{26}$ Hence, no player has relevant information on the deviator's identity: each player is either a neighbor of both $j$ and $k$, or a neighbor of none of them. Moreover, both deviations induce the same distribution over the messages received by player $i$, even when $\bar{\phi}$ is a mixed strategy. ${ }^{27}$

\footnotetext{
${ }^{26}$ These deviations of players $j$ and $k$ also induce the same distribution of payoffs for all players who are neighbors of both $j$ and $k$.

${ }^{27}$ Indeed, player $j$ draws a fictitious history, using $\bar{\phi}^{j}$. If $\bar{\phi}^{j}$ is mixed, so is $\psi^{j}$ (and similarly for player $k$ 's deviation).
} 
As a consequence, at every stage $t$, for each private history $h_{t}^{i}$ of player $i$ :

$$
\mathbb{P}_{\tau^{j}, \bar{\sigma}^{-j}, \psi^{j}, \bar{\phi}^{-j}}\left(h_{t}^{i}\right)=\mathbb{P}_{\tau^{k}, \bar{\sigma}^{-k}, \psi^{k}, \bar{\phi}^{-k}}\left(h_{t}^{i}\right) .
$$

Now, I define the following numbers $b_{t}$ and $c_{t}$ :

$$
\begin{aligned}
& b_{t}=\mathbb{P}_{\tau^{j}, \bar{\sigma}^{-j}, \psi^{j}, \bar{\phi}^{-j}}\left(a_{t}^{i}=C\right)=\mathbb{P}_{\tau^{k}, \bar{\sigma}^{-k}, \psi^{k}, \bar{\phi}^{-k}}\left(a_{t}^{i}=C\right), \\
& c_{t}=\mathbb{P}_{\tau^{j}, \bar{\sigma}^{-j}, \psi^{j}, \bar{\phi}^{-j}}\left(a_{t}^{i}=D\right)=\mathbb{P}_{\tau^{k}, \bar{\sigma}^{-k}, \psi^{k}, \bar{\phi}^{-k}}\left(a_{t}^{i}=D\right) .
\end{aligned}
$$

Under $\left(\tau^{j}, \psi^{j}, \bar{\sigma}^{-j}, \bar{\phi}^{-j}\right)$, player $j$ 's expected payoff at stage $t$ is then: $g_{t}^{j}\left(\tau^{j}, \bar{\sigma}^{\mathcal{N}(j)}\right) \geq 4 c_{t} \geq$ $4\left(1-b_{t}\right)$. As a consequence, $\gamma_{\delta}^{j}\left(\tau^{j}, \psi^{j}, \bar{\sigma}^{-j}, \bar{\phi}^{-j}\right) \geq 4(1-\delta) \sum_{t=1}^{+\infty} \delta^{t-1}\left(1-b_{t}\right)$. Since $(\bar{\sigma}, \bar{\phi})$ is a Nash equilibrium of the repeated game, there must exist $\bar{\delta} \in(0,1)$ such that for any $\delta \in(\bar{\delta}, 1), 4(1-\delta) \sum_{t=1}^{+\infty} \delta^{t-1}\left(1-b_{t}\right) \leq 1$, so that $(1-\delta) \sum_{t=1}^{+\infty} \delta^{t-1} b_{t} \geq \frac{3}{4}$. In the same way, player $k$ 's expected payoff under $\left(\tau^{k}, \psi^{k}, \bar{\sigma}^{-k}, \bar{\phi}^{-k}\right)$ at stage $t$ is: $g_{t}^{k}\left(\tau^{k}, \bar{\sigma}^{\mathcal{N}(k)}\right) \geq 4 b_{t}$. Hence, $\gamma_{\delta}^{k}\left(\tau^{k}, \psi^{k}, \bar{\sigma}^{-k}, \bar{\phi}^{-k}\right) \geq 4(1-\delta) \sum_{t=1}^{+\infty} \delta^{t-1} b_{t}$. However, there exists $\bar{\delta} \in(0,1)$ such that for any $\delta \in(\bar{\delta}, 1),(1-\delta) \sum_{t=1}^{+\infty} \delta^{t-1} b_{t} \geq \frac{3}{4}$, so $\gamma_{\delta}^{k}\left(\tau^{k}, \psi^{k}, \bar{\sigma}^{-k}, \bar{\phi}^{-k}\right) \geq 3$. This contradicts the fact that $(\bar{\sigma}, \bar{\phi})$ is a Nash equilibrium of the repeated game.

\section{A.2 Payoff function with more than two actions}

In this section, I modify the payoff function constructed in Section 6 for games in which players have more than two actions. For this purpose, I duplicate rows, columns, matrices... in the following manner. For each player $p \in N$, identify $A^{p}$ with $\left\{1, \ldots, k_{p}\right\}$, where $k_{p}=\sharp A^{p}$. The payoff functions are the following.

- For each player $m \neq i, j, k$ such that $m \notin \mathcal{N}(j) \cap \mathcal{N}(k)$, player $m$ 's payoff at stage $t$ is $g^{m}\left(a_{t}^{m}, a_{t}^{\mathcal{N}(m)}\right)=\ell_{t} \frac{\epsilon}{n}+\frac{1}{\sum_{p \in \mathcal{N}(m) \cup\{m\}} k_{p}}$.

- For each player $m \neq i, j, k$ such that $m \in \mathcal{N}(j) \cap \mathcal{N}(k)$, player $m$ 's payoff at stage $t$ is:

$$
g^{m}\left(a_{t}^{m}, a_{t}^{\mathcal{N}(m)}\right)= \begin{cases}1+\ell_{t}^{-j k} \frac{\epsilon}{n}+\frac{1}{\sum_{p \in \mathcal{N}(m) \backslash\{j, k\} \cup\{m\}} k_{p}} & \text { if } a^{j}=a^{k} \\ \ell_{t}^{-j k} \frac{\epsilon}{n}+\frac{1}{\sum_{p \in \mathcal{N}(m) \backslash\{j, k\} \cup\{m\}} k_{p}} & \text { otherwise. }\end{cases}
$$


- Player $i$ 's payoff at stage $t$ is:

$$
g^{i}\left(a_{t}^{i}, a_{t}^{\mathcal{N}(i)}\right)=\left\{\begin{array}{l}
u^{i}\left(a_{t}^{i}, a_{t}^{j}, a_{t}^{k}\right) \text { if } a_{t}^{p} \in\{1,2\} \text { for each } p \in \mathcal{N}(i), \\
u^{i}\left(a_{t}^{i}, a_{t}^{j}, a_{t}^{k}\right)-\frac{1}{\sum_{p \in \mathcal{N}(i) \cup\{i\}} k_{p}} \text { if } a_{t}^{i}, a_{t}^{j}, a_{t}^{k} \in\{1,2\}, a_{t}^{m} \geq 3 \forall m \in \mathcal{N}(i) \backslash\{i, j, k\} \\
6-\frac{1}{\sum_{p \in \mathcal{N}(i) \cup\{i\}} k_{p}} \text { otherwise. }
\end{array}\right.
$$

- For each player $n \in\{j, k\}$, player $n$ 's payoff at stage $t$ is:

$$
g^{n}\left(a_{t}^{n}, a_{t}^{\mathcal{N}(n)}\right)=\left\{\begin{array}{l}
u^{n}\left(a_{t}^{i}, a_{t}^{j}, a_{t}^{k}\right) \text { if } a_{t}^{p} \in\{1,2\} \text { for each } p \in \mathcal{N}(n), \\
u^{n}\left(a_{t}^{i}, a_{t}^{j}, a_{t}^{k}\right)+\frac{1}{\sum_{p \in \mathcal{N}(n) \cup\{n\}} k_{p}} \text { if } a_{t}^{i}, a_{t}^{j}, a_{t}^{k} \in\{1,2\}, a_{t}^{m} \geq 3 \forall m \neq i, j, k, \\
6+\frac{1}{\sum_{p \in \mathcal{N}(n) \cup\{n\}} k_{p}} \text { otherwise. }
\end{array}\right.
$$

It is easy to see that this payoff function satisfies Assumption $\mathrm{P}$, and that all the desired properties for the proof of necessity in Section 6 hold.

\section{B Proof of Lemma 5.2}

Proof. Take a network $G$ that satisfies Condition DN and a player $k \in N$ such that $\sharp \mathcal{N}(k) \geq$ 2. Assume that player $k$ deviates in action at stage $t$. Take any player $j \neq k$. I first prove that $j$ is cleared at stage $t+1$ by every player $i \in N$. Two cases are possible.

(1) Assume first that for each player $j^{1} \in \mathcal{N}(j), \sharp \mathcal{N}\left(j^{1}\right) \geq 2$. Then, each player $i$ in $N$ clears player $j$ at stage $t+1$. Indeed, the following holds:

- first, if $j$ plays $\phi^{* j}$ at stage $t+1$, then $j \in m_{t+1}^{j}(N)$. Indeed, either $j \in \mathcal{N}(k)$ and $\tilde{\phi}^{j}$ prescribes player $j$ to announce publicly $N \backslash \mathcal{N}(j)$ to all players at stage $t+1$, so $j \in \tilde{m}_{t+1}^{j}(N)$. Or $j \notin \mathcal{N}(k)$ and player $j$ starts the protocol at the end of stage $t+1,{ }^{28}$ so $j$ is prescribed to announce $\mathcal{N}(j) \cup\{j\}$ publicly to all players at stage $t+1$, so $j \in \bar{m}_{t+1}^{j}(N)$.

- Second, since Condition DN is satisfied, there exists a player $m \neq j, k$ such that $m \in$ $\mathcal{N}(k) \backslash\{j\} \triangle \mathcal{N}(j) \backslash\{k\}$. Moreover, if $m$ plays $\phi^{* m}$ at stage $t+1$, then $j \in m_{t+1}^{m}(N)$.

\footnotetext{
${ }^{28}$ Notice that player $j$ could have started the protocol at the end of stage $t$ if (a) one of his neighbor deviated at stage $t$, or (b) if a player who is not his neighbor deviated at stage $t-1$, or (c) if player $k$ deviated also in communication at stage $t$ by sending to $j$ a message different from $\bar{m}_{t+1}^{k}(N)$. However, in any case, player $j$ ends this "previous" protocol at the end of stage $t$, and starts a "new" protocol at the end of stage $t+1$.
} 
Indeed, either, $m \in \mathcal{N}(k)$ and $\tilde{\phi}^{m}$ prescribes player $m$ to announce $N \backslash \mathcal{N}(m)$ publicly to all players at stage $t+1$, so $j \in \tilde{m}_{t+1}^{m}(N)$. Or $m \notin \mathcal{N}(k)$, and $\bar{\phi}^{m}$ prescribes player $m$ to announce $\mathcal{N}(m) \cup\{m\}$ publicly to all players at stage $t+1$, so $j \in \bar{\phi}^{m}(N)$.

- Third, if $k$ follows $\phi^{* k}$ at stage $t+1$, then $\tilde{\phi}^{k}$ prescribes player $k$ to announce $N \backslash\{k\}$ publicly to all players at stage $t+1$, so $j \in \tilde{m}_{t+1}^{k}(N)$.

Since at most one player in $\{j, k, m\}$ deviates in communication at stage $t+1$, then $j \notin X_{t+1}^{i}$ for each player $i \in N$.

(2) Assume now that there exists $j^{1} \in \mathcal{N}(j)$ such that $\mathcal{N}\left(j^{1}\right)=\{j\}$. First, $j^{1}$ is unique (see Remark 4.2). Second, $G$ is connected and $n \geq 3$, so $\sharp \mathcal{N}(j) \geq 2$. Finally, $j^{1} \neq k$ since $\sharp \mathcal{N}(k) \geq 2$ by assumption. Hence, $j^{1}$ is cleared at stage $t+1$ by every player $i$ in $N$ (see (1) above). In addition, with the same reasoning as before, at least two players in $\{j, k, m\}$ make public announcements including $j$ to all players at stage $t+1$. As a consequence, for each player $i \in N, j \notin X_{t+1}^{i}$.

Finally, I prove that no player $i \in N$ clears player $k$, i.e. $k \in X_{t+1}^{i}$ for each player $i \in N$. By construction, $k \notin m_{t+1}^{j}(N)$ for any $j \in N$ who follows $\phi^{* j}$. Since at most one player deviates in communication at stage $t+1, k \in X_{t+1}^{i}$ for each player $i \in N$.

Hence, $X_{t+1}^{i}=\{k\}$ for each player $i$ in $N$.

\section{Proof of Lemma 5.3}

Proof. Take a connected network $G$ that satisfies Condition DN and suppose Assumption P holds. Take a pair of players $k$ and $\ell$ such that $\mathcal{N}(k)=\{\ell\}$.

Assume first that player $k$ deviates in action at stage $t$. At stage $t+1$, players $k$ and $\ell$ should announce $N^{-k}$ and $N \backslash \mathcal{N}(k)$ respectively publicly to all players. All other players $j \neq k, \ell$ should announce $\mathcal{N}(j) \cup\{j\}$ and $k \notin m_{t+1}^{j}(N)$ publicly to all players since $\mathcal{N}(k)=$ $\{\ell\}$. As a consequence, the name of $k$ appears in at most one public announcement at stage $t$ and $X_{t+1}^{i} \supseteq\{k, \ell\}$ for each player $i$ in $N$. In addition, no player $j \neq k$, including player $\ell$, deviates in action at stage $t$ under unilateral deviations. So, each player $j \neq k$ such that $\sharp \mathcal{N}(j) \geq 2$ appears in the public announcements of at least two distinct players (among his two neighbors and himself), and $j \notin X_{t+1}^{i}$ for each player $i \in N$. On the other hand, each player $j \neq k$ such that $\sharp \mathcal{N}(j)=1$ also appears in the public announcements of at least two distinct players: either $j$ and his single neighbor do not deviate and $j$ appears in both of their public announcements, or one of them deviates at stage $t+1$, which implies that $k$ follows 
$\phi_{t+1}^{* k}$ and $j \in m_{t+1}^{k}(N)$. As a consequence, each player $j \neq k$ is cleared by all players at stage $t+1$. Hence, $X_{t+1}^{i}=\{k, \ell\}$ for each player $i$ in $N$.

Assume now that there is no action deviation at stage $t$ and that $m_{t+1}^{k}=N^{-k}$. Player $k$ thus deviates in communication at stage $t+1$ and no other player does under unilateral deviations. It follows that all other players $j \neq k$ announce $\mathcal{N}(j) \cup\{j\}$ publicly since there was no action deviation at stage $t$. As a consequence, each player $j \neq k$ is cleared by at least two players at stage $t+1$, and player $k$ appears in the public announcement of player $\ell$ only. Henceforth, $X_{t+1}^{i}=\{k, \ell\}$ for each player $i \in N$.

Finally, assume that there is no action deviation at stage $t$ and that $m_{t+1}^{\ell}(N)=N \backslash \mathcal{N}(\ell)$. Player $\ell$ thus deviates in communication at stage $t+1$ and no other player does under unilateral deviations. It follows that all other players $j \neq k$ announce $\mathcal{N}(j) \cup\{j\}$ publicly to all players since there was no action deviation at stage $t$. As a consequence, each player $j \notin \mathcal{N}(\ell)$ is cleared by at least two players at stage $t+1$. Moreover, for each player $j \in$ $\mathcal{N}(\ell) \backslash\{k\}, \sharp \mathcal{N}(j) \geq 2$. Indeed, if it was not the case, then $\mathcal{N}(j)=\{\ell\}=\mathcal{N}(k)$ which contradicts Condition DN. Therefore, each player $j \in \mathcal{N}(\ell) \backslash\{k\}$ also appears in the public announcements of at least two distinct players at stage $t+1$. Finally, player $k$ appears in his own public announcement only. As a conclusion, $X_{t+1}^{i}=\{k, \ell\}$ for every player $i$ in $N$.

\section{Proof of Lemma 5.4}

Proof. Take a connected network $G$ that satisfies Condition DN, and suppose that Assumption $\mathrm{P}$ holds. Take a player $k$ in $N$ and assume that there is no action deviation at stage $t$.

Assume first that $\mathcal{N}(k)=\{\ell\}$ and that $k$ deviates in communication at stage $t+1$. If $m_{t+1}^{k} \neq m_{t+1}^{k}(N)$ ( $k$ does not make a public announcement to all players), then the message of player $k$ is ignored. Assume now that $m_{t+1}^{k}=m_{t+1}^{k}(N)$ and $\left\{N^{-k}, \mathcal{N}(k) \cup\{k\}\right\} \notin m_{t+1}^{k}(N)$. Under unilateral deviations, all other players $j \neq k$ announce $\mathcal{N}(j) \cup\{j\}$ publicly. Two cases are then possible. Either $N \backslash \mathcal{N}(k) \notin m_{t+1}^{k}(N)$ and $m_{t+1}^{k}(N)$ is not taken into account under $\phi^{*}$ since it implies that player $k$ 's message is different from the kinds of messages regarded during the communication protocol. Then, $X_{t+1}^{i}=\emptyset$ for every player $i$ in $N$. Or, $N \backslash \mathcal{N}(k) \in m_{t+1}^{k}(N)$. Player $k$ is then cleared by all players since his name is in the public announcements of $k$ and $\ell$. Player $\ell$ is also cleared by all players because his name is in at least two public announcements among his own and his other neighbor than $k$ (since $n \geq 3$ and $G$ is connected, $\sharp \mathcal{N}(k) \geq 2$ ). Each other player $i$ is cleared by all players since his name appears in the public announcements of at least two players among him and his neighbors (each has at least one neighbor since $G$ is connected). Hence, $X_{t+1}^{i}=\emptyset$ for every player $i$ in 
$N$.

Assume now that there exists $k^{\prime}$ such that $\mathcal{N}\left(k^{\prime}\right)=k$, and that $k$ deviates in communication at stage $t+1$. If $m_{t+1}^{k} \neq m_{t+1}^{k}(N)$, then the message of player $k$ is ignored. Assume now that $m_{t+1}^{k}=m_{t+1}^{k}(N)$ and that $\{N \backslash \mathcal{N}(k), \mathcal{N}(k) \cup\{k\}\} \notin m_{t+1}^{k}(N)$. Under unilateral deviations, all other players $j \neq k$ announce $\mathcal{N}(j) \cup\{j\}$ publicly to all players. Two cases are possible. Either $N^{-k} \notin m_{t+1}^{k}(N)$ and player $k$ 's public announcement is not taken into account as before. Or $N^{-k} \in m_{t+1}^{k}(N)$, in which case it is obvious that all players are cleared (recall that player $k$ has at least two neighbors). In any case, $X_{t+1}^{i}=\emptyset$ for each player $i$ in $N$.

Finally, assume $\sharp \mathcal{N}(k) \geq 2, \sharp \mathcal{N}(j) \geq 2$ for every $j \in \mathcal{N}(k)$, and that $k$ deviates in communication at stage $t+1$. If $m_{t+1}^{k} \neq m_{t+1}^{k}(N)$, then player $k$ 's message is ignored. Assume now $m_{t+1}^{k}=m_{t+1}^{k}(N)$ and $\{\mathcal{N}(k) \cup\{k\}\} \notin m_{t+1}^{k}(N)$. Under unilateral deviations, all other players $j \neq k$ announce $\mathcal{N}(j) \cup\{j\}$ publicly to all players. Three cases are possible. Assume first that $N^{-k} \in m_{t+1}^{k}(N)$. Then all players are cleared by everybody at stage $t+1$, since player $k$ has at least two neighbors. Second, assume $N \backslash \mathcal{N}(k) \in m_{t+1}^{k}(N)$. Since player $k$ 's neighbors have more than two neighbors, they are cleared by all players at stage $t+1$. Obviously, so are players other than $k$ 's neighbors. Third, assume $\left\{N^{-k}, N \backslash \mathcal{N}(k)\right\} \notin$

$m_{t+1}^{k}(N)$. As before, $m_{t+1}^{k}$ is not taken into account. Therefore, in any case, $X_{t+1}^{i}=\emptyset$ for each player $i$ in $N$.

\section{References}

[1] Abreu, D., Dutta, P., And Smith, L. The folk theorem for repeated games: a neu condition. Econometrica: Journal of the Econometric Society (1994), 939-948.

[2] Ashrenazi-Golan, G. Confession and pardon in repeated games with private monitoring and communication.

[3] Aumann, R., Maschler, M., And Stearns, R. Repeated Games with Incomplete Information. The MIT press, 1995.

[4] Aumann, R. J., And Shapley, L. S. Long term competition? a game theoretic analysis. Unpublished; reprinted in: Aumann R.J., Collected Papers, Volume 1 (1976).

[5] Ben-Porath, E., And Kahneman, M. Communication in repeated games with private monitoring. Journal of Economic Theory 70, 2 (1996), 281-297.

[6] Bramoullé, Y., And Kranton, R. Public goods in networks. Journal of Economic Theory 135, 1 (2007), 478-494. 
[7] CHo, M. Cooperation in the repeated prisoner's dilemma game with local interaction and local communication. Unpublished manuscript.

[8] Compte, O. Communication in repeated games with imperfect private monitoring. Econometrica 66, 3 (1998), 597-626.

[9] Diestel, R. Graph theory, graduate texts in mathematics vol. 173, 2000.

[10] Fudenberg, D., And Levine, D. K. An approximate folk theorem with imperfect private information. Journal of Economic Theory 54, 1 (1991), 26-47.

[11] Fudenberg, D., Levine, D. K., And Maskin, E. The folk theorem with imperfect public information. Econometrica (1994), 997-1039.

[12] Fudenberg, D., And Maskin, E. The folk theorem in repeated games with discounting or with incomplete information. Econometrica 54, 3 (1986), 533-554.

[13] Fudenberg, D., And Maskin, E. On the dispensability of public randomization in discounted repeated games. Journal of Economic Theory 53, 2 (1991), 428-438.

[14] Fudenberg, D., And Tirole, J. Perfect bayesian equilibrium and sequential equilibrium. Journal of Economic Theory 53, 2 (1991), 236-260.

[15] Galeotti, A., Goyal, S., Jackson, M. O., Vega-Redondo, F., and Yariv, L. Network games. Review of Economic Studies 7r7, 1 (2010), 218-244.

[16] González-Díaz, J., And Meléndez-Jiménez, M. On the notion of perfect bayesian equilibrium. TOP (2011), 1-16.

[17] Goyal, S. Connections: an Introduction to the Economics of Networks. Princeton University Press, 2009.

[18] Green, E. J., And Porter, R. H. Noncooperative collusion under imperfect price information. Econometrica (1984), 87-100.

[19] Hotelling, H. Stability in competition. The Economic Journal 39, 153 (1929), 41-57.

[20] Jackson, M. O. Social and Economic Networks. Princeton Univ Press, 2008.

[21] Kandori, M., And Matsushima, H. Private observation, communication and collusion. Econometrica 66, 3 (1998), 627-652.

[22] Kinateder, M. Repeated games played in a network. Unpublished manuscript. 
[23] LaClau, M. A folk theorem for repeated games played on a network. Games and Economic Behavior 76, 2 (2012), 711-737.

[24] LeHrer, E. Lower equilibrium payoffs in two-player repeated games with nonobservable actions. International Journal of Game Theory 18, 1 (1989), 57-89.

[25] Mailath, G. J., And Samuelson, L. Repeated Games and Reputations: Long-Run Relationships. Oxford University Press, USA, 2006.

[26] Nava, F., And Piccione, M. Repeated games with local monitoring. Unpublished manuscript.

[27] Obara, I. Folk theorem with communication. Journal of Economic Theory 144, 1 (2009), 120-134.

[28] RADnER, R. Repeated partnership games with imperfect monitoring and no discounting. The Review of Economic Studies 53, 1 (1986), 43-57.

[29] Renault, J., And Tomala, T. Repeated proximity games. International Journal of Game Theory 27, 4 (1998), 539-559.

[30] Renault, J., And Tomala, T. Communication equilibrium payoffs in repeated games with imperfect monitoring. Games and Economic Behavior 49, 2 (2004), 313-344.

[31] Rubinstein, A. Equilibrium in supergames. Research Memorandum 25 (1977), 1-9.

[32] Sorin, S. On repeated games with complete information. Mathematics of Operations Research 11, 1 (1986), 147-160.

[33] Sorin, S. Repeated games with complete information. in Handbook of game theory with economic applications, Aumann, R.J. and Hart, S. (eds) 1 (1992), 71-107.

[34] Tomala, T. Fault reporting in partially known networks and folk theorems. Operations Research 59, 3 (2011), 754-763.

[35] Wolitzky, A. Cooperation with network monitoring. forthcoming in Review of Economic Studies. 